\title{
11. Inhaltliche Ausgestaltung und Leitungsfunktionen im freiwilligen Engagement
}

\author{
Christine Hagen \& Julia Simonson
}

\section{Kernaussagen}

Mit dem Engagement sind unterschiedliche Tätigkeitsinhalte verknüpft. Dabei dominieren praktisch-organisatorische Tätigkeiten. Die Geschlechterunterschiede sind insgesamt gering: So gehen Männer eher als Frauen administrativen Tätigkeiten nach, Frauen leisten hingegen vergleichsweise häufiger persönliche Hilfeleistungen.

Die Aufgaben der freiwillig Engagierten werden vielfältiger. Im Zeitverlauf seit 1999 hat die Tätigkeitsdichte kontinuierlich zugenommen und es lässt sich eine größere Vielfalt von Inhalten beobachten, mit denen sich Engagierte im Rahmen ihrer freiwilligen Tätigkeit auseinandersetzen.

Leitungs- und Vorstandsfunktionen im Engagement sind verbreitet. Über ein Viertel aller Engagierten hat eine Leitungs- oder Vorstandsfunktion, wobei im Jahr 2014 Männer immer noch deutlich häufiger eine Leitungs- und Vorstandstätigkeit ausüben als Frauen. Anteilig an allen Engagierten nimmt die Leitungs- und Vorstandstätigkeit seit fünfzehn Jahren kontinuierlich ab, dies ist jedoch auf eine starke Zunahme der Zahl von Engagierten ohne Leitungs- und Vorstandsfunktion zurückzuführen.

Über die Hälfte der Engagierten nutzt das Internet für ihre Tätigkeit. Insbesondere bei den älteren Engagierten wird das Internet häufiger von Männern als von Frauen genutzt. Sich überwiegend oder sogar ausschließlich über das Internet zu engagieren, ist allerdings äußerst selten.

Unterschiedliche Personen engagieren sich für unterschiedliche Zielgruppen. Die am häufigsten genannte Zielgruppe des freiwilligen Engagements sind Kinder und Jugendliche. Insgesamt engagiert sich knapp die Hälfte der Engagierten für diese Personengruppe, von den jüngeren Engagierten sind es sogar zwei Drittel. Freiwilliges Engagement, das sich an ältere Menschen, Menschen mit Behinderungen sowie an finanziell oder sozial schlechter gestellte Menschen richtet, wird hingegen häufiger von Älteren als von Jüngeren und dabei häufiger von älteren Frauen als von älteren Männern ausgeübt.

Engagement hat einen lokalen Bezug. Das freiwillige Engagement in Deutschland richtet sich in erster Linie auf die Wohnregion; Engagement mit Bezug auf eine andere Region in Deutschland, in Europa oder außerhalb Europas findet vergleichsweise seltener statt. 


\subsection{Einleitung}

Freiwilliges Engagement ist vielfältig: In ganz unterschiedlichen gesellschaftlichen Bereichen üben Personen freiwillig und unentgeltlich Tätigkeiten aus, die mit verschiedenen Inhalten und Aufgaben verbunden sind und die unterschiedlichen Zielgruppen zugutekommen. Dieses Kapitel gibt Auskunft über verschiedene Aspekte der inhaltlichen Ausgestaltung der freiwilligen Tätigkeit. Gefragt wird also: Wer engagiert sich wie und für wen?

Mit den Angaben der Engagierten zu den Inhalten ihrer freiwilligen Tätigkeiten ist es im Freiwilligensurvey möglich, einen Überblick über die Aufgaben und Anforderungen im Engagement zu geben. Auch wenn die Bereiche, in denen sich Personen engagieren, ganz unterschiedlich sein können, können sich die Tätigkeiten und ihre Anforderungen ähneln. Andererseits können auch in demselben gesellschaftlichen Bereich ganz unterschiedliche freiwillige Tätigkeiten ausgeübt werden. Bekannt ist, dass sich Männer und Frauen häufig in unterschiedlichen Bereichen und mit anderen Tätigkeitsschwerpunkten engagieren. Bisherige Ergebnisse des Freiwilligensurveys zeigen, dass Frauen stärker in Bereichen mit betreuenden, gesundheitlichen, helfenden und versorgenden Aufgaben freiwillig tätig waren und Männer sich stärker in Bereichen engagierten, die mehr freizeit- und bildungsorientiert beziehungsweise politisch $\mathrm{zu}$ verorten sind (Gensicke 2015). Unterschiede in den konkreten Tätigkeitsinhalten zeigten sich beispielsweise bei den persönlichen Hilfeleistungen, die häufiger von Frauen erbracht wurden, und bei Verwaltungstätigkeiten, die zu größeren Anteilen von Männern ausgeübt wurden (Gensicke, Picot \& Geiss 2006). Ein Augenmerk soll daher auf geschlechtsspezifische Unterschiede hinsichtlich der Ausgestaltung des Engagements gelegt werden.

Untersucht wird ferner die Entwicklung der ehrenamtlichen Leitungs- und Vorstandstätigkeiten. Einer Organisationsbefragung zufolge geben 85 Prozent der Vereine an, dass es für sie zunehmend schwieriger wird, diese ehrenamtlichen Funktionen zu besetzen (Priller, Alscher, Droß, Paul, Poldrack, Schmeißer \& Waitkus 2012; Alscher, Droß, Priller \& Schmeißer 2013). Große Organisationen wie der Deutsche Olympische Sportbund (DOSB) suchen vermehrt nach neuen Möglichkeiten wie der Ansprache neuer Zielgruppen, um ausreichend Ehrenamtliche für die Übernahme von Funtkionen zu gewinnen und dauerhaft $\mathrm{zu}$ binden (Blessing-Kapelke \& Kaiser 2013). Auch auf der Grundlage der bisherigen Erhebungen des Freiwilligensurveys hat sich gezeigt, dass der Anteil der Engagierten mit Leitungs- oder Vorstandstätigkeiten im Zeitverlauf zurückgegangen ist (Gensicke 2015: 165). Es ist zu fragen, ob sich dieser Trend fortsetzt und wie er $\mathrm{zu}$ bewerten ist. Auch hier werden geschlechtsspezifische Unterschiede betrachtet: Üben Männer wie in den Untersuchungsjahren zuvor häufiger eine Leitungs- und Vorstandsfunktion aus als Frauen oder hat hier möglicherweise im Zeitverlauf eine Angleichung zwischen Frauen und Männern stattgefunden? Darüber hinaus wird über die Leitungs- und Vorstandsfunktionen in den unterschiedlichen Engagementbereichen berichtet. Es ist $\mathrm{zu}$ vermuten, dass insbesondere Bereiche mit eher institutionalisierten Organisationen wie beispielsweise die berufliche oder politische Interessenvertretung sich nach wie vor durch eher hohe Anteile von Leitungs- oder Vorstandstätigkeiten auszeichnen (Gensicke 2015: 165; von Rosenbladt 2001: 75), auch da jeder Verein verpflichtend solche Positionen zu besetzen hat.

Veränderungen der freiwilligen Tätigkeiten ergeben sich auch durch den technischen Fortschritt - mit der zunehmenden Verbreitung des Internets als Kommunikations- und Arbeitsmittel stellt sich die Frage, welche Bedeutung das Internet im freiwilligen Engagement hat. In welchem Ausmaß lässt sich eine neue Form des Engagements beobachten, welches überwiegend oder sogar ausschließlich über das Internet ausgeübt wird, sogenanntes Online-Volunteering? 
Online-Volunteering zeichnet sich laut Jähnert (2012) dadurch aus, dass es, neben den sonstigen in Anlehnung an die Enquete-Kommission ,Zukunft des Bürgerschaftlichen Engagements (2002: 38ff.) vorgebrachten Bestimmungskriterien, orts- und zeitunabhängig ist. Das Internet bietet also die Möglichkeit, sich außerhalb eines Vereins oder einer sonstigen Organisation auch von zu Hause aus oder von unterwegs aus zu engagieren, beispielsweise wenn eine Internetplattform zum Meinungsaustausch oder als Informationsportal betrieben wird. Zwar ist orts- und zeitunabhängiges Engagement auch ohne Nutzung des Internets denkbar, beispielsweise, wenn Beratungsleistungen von zu Hause aus über das Telefon erbracht werden; das Internet vereinfacht diese Form des freiwilligen Engagements jedoch deutlich.

Freiwilliges Engagement kann unterschiedlichen Personengruppen zugutekommen. Im Freiwilligensurvey wird daher gefragt, an welche Zielgruppen sich das freiwillige Engagement richtet. Als häufigste Zielgruppe wurden in der Vergangenheit durchgängig Kinder und Jugendliche genannt. Die Zielgruppe der älteren Menschen war demgegenüber deutlich kleiner, hat jedoch im Zeitverlauf leicht an Bedeutung gewonnen (Gensicke 2015). Aufgrund des demografischen Wandels ist zu erwarten, dass Ältere eine zunehmend wichtigere Zielgruppe im Engagement werden. Sie stellen eine bedeutende und größer werdende Adressatengruppe freiwilligen Engagements dar. Darüber hinaus stellt sich auch die Frage, wer sich für wen engagiert. Hagen und Vogel (2012) konnten zeigen, dass sich jüngere Frauen und Männer in erster Linie für ihre eigene Altersgruppe oder noch Jüngere engagieren. Auch im mittleren Lebensalter spielt das Engagement für Kinder und Jugendliche noch eine bedeutende Rolle. In höherem Alter steht dann sowohl für Frauen als auch für Männer das Engagement für andere ältere Menschen im Vordergrund. Es gibt also sowohl Engagement innerhalb der Generationen als auch generationenübergreifendes Engagement. $\mathrm{Zu}$ fragen ist, ob diese Muster weiterhin Bestand haben oder generationenübergreifendes Engagement im Zuge des demografischen Wandels im Zeitverlauf zunimmt, sich also beispielsweise auch jüngere Menschen und Personen im mittleren Lebensalter häufiger für Ältere engagieren.

Vor dem Hintergrund der im Jahr 2015 steigenden Zahl der aus Krisengebieten nach Deutschland kommenden Menschen hat das Engagement für Flüchtlinge stark an Bedeutung gewonnen (Karakayali \& Kleist 2015; Eisnecker \& Schupp 2016). Mit den Daten des Freiwilligensurveys lässt sich diese Entwicklung allerdings nicht nachzeichnen, da viele Initiativen zur Unterstützung von Flüchtlingen erst nach der Erhebung des Freiwilligensurveys 2014 ihre Arbeit aufnahmen beziehungsweise ausbauten. Darüber hinaus wird im Freiwilligensurvey zwar Engagement erfasst, das Menschen mit Migrationshintergrund zugutekommt; dabei kann es sich aber auch um andere Einwanderergruppen wie zum Beispiel Arbeitsmigrantinnen und -migranten handeln.

Freiwilliges Engagement kann sich nicht nur an unterschiedliche Gruppen, sondern auch auf verschiedene Orte oder Regionen richten. Moderne Transport- und Kommunikationsmittel ermöglichen das Engagement auch über den eigenen Wohn- und Aufenthaltsort hinaus. Daher wird untersucht, inwieweit das Engagement hauptsächlich der unmittelbaren Wohnregion zugutekommt oder sich das Engagement auf andere Regionen in Deutschland, Europa oder einem Land außerhalb Europas richtet.

Konkret werden in diesem Kapitel folgende Forschungsfragen untersucht:

1. Welche Tätigkeitsinhalte sind mit dem freiwilligen Engagement verbunden? Wie haben sich diese im Zeitverlauf in verschiedenen Bevölkerungsgruppen verändert?

2. Wie hoch ist der Anteil von Personen mit einer Leitungs- oder Vorstandstätigkeit in verschiedenen Bevölkerungsgruppen und in den unterschiedlichen Engagementbereichen? Wie hat sich der Anteil von Engagierten mit 
Leitungs- oder Vorstandsfunktion im Zeitverlauf verändert?

3. Wie hoch ist der Anteil derer, die im freiwilligen Engagement das Internet nutzen? Wie hoch ist der Anteil an Engagierten, die ihr En- gagement ausschließlich oder überwiegend im Internet ausüben?

4. An welche Zielgruppen und auf welche Zielregionen richtet sich das freiwillige Engagement von verschiedenen Bevölkerungsgruppen?

\subsection{Datengrundlage}

Die in diesem Kapitel berichteten Befunde basieren auf gewichteten Analysen. Für die Gewichtung wurden in allen Erhebungsjahren dieselben Gewichtungsmerkmale berücksichtigt. Dabei handelt es sich um die Designgewichtung, die sich auf die Auswahlwahrscheinlichkeit einer Person bezieht (Haushaltsgröße, Zahl der Festnetznummern und, nur für das Jahr 2014, auch die Zahl der Mobilfunknummern) und Gewichtungsmerkmale, die Abweichungen der Stichprobenverteilung von der Grundgesamtheit in bestimmten Dimensionen ausgleichen (Bundesland, Gemeindegrößenklassen, Geschlecht, Altersgruppen). Von einer Hinzunahme weiterer Gewichtungsmerkmale wie etwa des Bildungsstandes haben wir abgesehen, um die Vergleichbarkeit mit den bisher veröffentlichten Berichten zu erhalten.

In diesem Kapitel stehen die Engagierten im Mittelpunkt. Alle Angaben beziehen sich auf die freiwillige Tätigkeit einer engagierten Person oder auf die zeitintensivste freiwillige Tätigkeit bei Mehrfachengagierten. Im Folgenden berichten wir Ergebnisse der standardisierten Abfrage der Tätigkeitsinhalte bezogen auf diese einzige oder zeitintensivste Tätigkeit. Die Frageformulierung (unter der Ermöglichung von Mehrfachnennungen) lautete in allen vier Erhebungen unverändert:

Was ist der Hauptinhalt Ihrer eigenen Tätigkeit [Tätigkeit einblenden]: Geht es überwiegend um persönliche Hilfeleistungen; die Organisation und Durchführung von Hilfsprojekten; die Organisation und Durchführung von Treffen oder Veranstal- tungen; Beratung; pädagogische Betreuung oder die Anleitung einer Gruppe; Interessenvertretung und Mitsprache; Informations- und Öffentlichkeitsarbeit; Verwaltungstätigkeiten; praktische Arbeiten, die geleistet werden müssen; Vernetzungsarbeit; Mittelbeschaffung, Fundraising; etwas anderes?

Antwortkategorien jeweils: ja; nein

Dabei wurde jeweils die von den Befragten gegebene offene Angabe zur freiwilligen Tätigkeit eingeblendet und vorgelesen.

Die Nutzung des Internets im freiwilligen Engagement wird im Freiwilligensurvey 2014 folgendermaßen erfragt:

Nutzen Sie für Ihre Tätigkeit [Tätigkeit einblenden] das Internet?

Antwortkategorien: ja; nein

Darüber hinaus wird erfasst, ob dabei eine interaktive Internetnutzung, zum Beispiel über die Beteiligung an Blogs oder in sozialen Netzwerken, eine Rolle spielt. Die entsprechende Frage lautet:

Nutzen Sie für diese Tätigkeit auch soziale Netzwerke oder beteiligen sich an Blogs, Foren oder Wikis?

Antwortkategorien: ja; nein 
Schließlich wird erhoben, ob die Tätigkeit überwiegend oder sogar ausschließlich über das Internet ausgeübt wird, sodass der Verbreitung von Online-Volunteering nachgegangen werden kann:

Findet Ihre Tätigkeit ausschließlich oder überwiegend oder nur teilweise im Internet statt?

Antwortkategorien: ausschließlich; überwiegend; nur teilweise

Die Information, ob Personen im Rahmen ihrer freiwilligen Tätigkeit eine Leitungs- oder Vorstandsfunktion haben, entstammt folgender Frage:

Haben Sie eine Leitungs- oder Vorstandsfunktion?

Antwortkategorien: ja; nein

Die Information, an welche Zielgruppe(n) sich die freiwillige Tätigkeit richtet, wird (unter der Ermöglichung von Mehrfachnennungen) wie folgt erhoben:

Ich lese Ihnen nun einige Personengruppen vor. Sagen Sie mir bitte, ob sich Ihre Tätigkeit speziell an eine oder mehrere dieser Gruppen richtet. Richtet sie sich speziell an Kinder und Jugendliche; speziell an Familien; speziell an ältere Menschen; speziell an Menschen mit Behinderung; speziell an Menschen mit Migrationshintergrund; speziell an Frauen; speziell an Männer; speziell an finanziell oder sozial schlechter gestellte Menschen; speziell an hilfe-oder pflegebedürftige Menschen; an eine andere Zielgruppe?

Antwortkategorien jeweils: ja; nein

Gibt eine Person an, dass sich die Tätigkeit an Kinder und Jugendliche richtet, so wird noch einmal nachgefragt:
Handelt es sich dabei überwiegend um Kleinkinder bis unter 6 Jahre; Schulkinder von 6 bis unter 14 Jahre; Jugendliche ab 14 Jahren; oder ist das Alter gemischt?

Auch bei der Angabe der Zielgruppe ,ältere Menschen' wird noch einmal nachgefragt. Zum einen wird eine genauere Eingrenzung der Altersgruppe vorgenommen:

Gehören diese Menschen vorwiegend folgenden Altersgruppen an: bis unter 75 Jahre; 75 Jahre und älter; oder ist das Alter gemischt?

Zum anderen wird gefragt, ob es sich überwiegend um Menschen handelt, die an Demenz erkrankt sind:

Geht es dabei überwiegend um Menschen, die an Demenz erkrankt sind?

Antwortkategorien: ja; nein

Neben der Zielgruppe wird im Freiwilligensurvey 2014 auch erfasst, auf welche Region sich das Engagement richtet:

Welcher Region kommt Ihre Tätigkeit hauptsächlich zugute? Ist es eher Ihre Wohnregion; eine andere Region in Deutschland; eine Region in Europa; oder eine Region außerhalb Europas?

Wird angegeben, dass die Tätigkeit einer Region außerhalb Europas zugutekommt, wird noch einmal nachgefragt:

Handelt es sich dabei um ein Entwicklungsland?

Antwortkategorien: ja; teils/teils; nein 


\subsection{Tätigkeitsinhalte im freiwilligen Engagement}

Die Inhalte der freiwilligen Tätigkeiten sind sehr vielfältig und unterschiedlich. Um eine bessere Übersicht über die verschiedenen Aufgabenin- halte zu bekommen, haben wir die im Freiwilligensurvey abgefragten Tätigkeitsinhalte in vier Inhaltsfelder untergliedert (Abbildung 11-1).

\section{Abbildung 11-1: Übersicht zu den Inhalten der freiwilligen Tätigkeit}

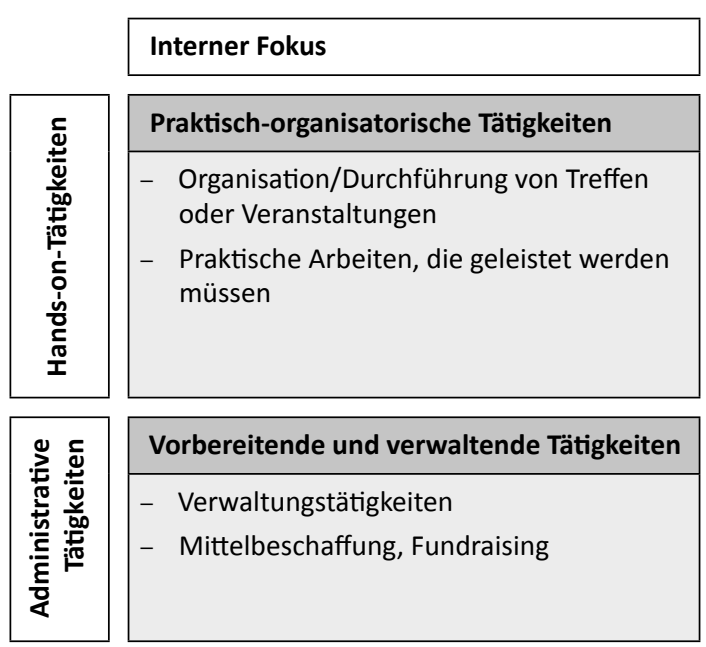

Quelle: Eigene Darstellung (DZA).

Hierzu werden zwei Dimensionen unterschieden: Die erste Dimension trennt die eher praktischen Aufgaben (Hands-on-Tätigkeiten) von eher verwaltungs-organisatorischen Aufgaben (administrative Tätigkeiten). Mit der zweiten Dimension wird der Fokus der Tätigkeitsinhalte beschrieben: Dieser kann entweder stärker auf die Organisation selbst (interner Fokus) oder aber nach außen gerichtet sein (externer Fokus). Die Kombination der beiden Dimensionen ermöglicht es, vier Inhaltsfelder zu unterscheiden: Praktisch-organisatorische Tätigkeiten umfassen die ,Organisation/Durchführung von Treffen oder Veranstaltungen' sowie ,Praktische Arbeiten, die geleistet werden müssen. Dies können beispielsweise Tätigkeiten wie die Vor- und Nachbereitung von Vereinssitzungen und Feierlichkeiten oder auch die Übernahme von Wegediensten im Kleingartenverein sein.

\section{Externer Fokus}

Helfende, beratende und betreuende Tätigkeiten

- Persönliche Hilfeleistungen

- Beratung

- Pädagogische Betreuung oder Anleitung einer Gruppe

Organisation/Durchführung von Hilfsprojekten

Öffentlichkeitsarbeit und Interessenvertretung

- Informations- und Öffentlichkeitsarbeit

- Interessenvertretung und Mitsprache

- Vernetzungsarbeit

Als helfende, beratende und betreuende Tätigkeiten werden ,Persönliche Hilfeleistungen', ,Beratung, ,Pädagogische Betreuung oder Anleitung einer Gruppe sowie die ,Organisation/ Durchführung von Hilfsprojekten 'verstanden. Gemeinsam ist diesen Tätigkeiten, dass sie sich jeweils auf eine mehr oder minder klar umrissene Zielgruppe wie beispielsweise Kinder und Jugendliche oder hilfebedürftige Menschen richten.

Vorbereitende und verwaltende Tätigkeiten umfassen ,Verwaltungstätigkeiten' sowie ,Mittelbeschaffung, Fundraising und damit Aufgaben, die für die Arbeit der jeweiligen Organisation oder Gruppe essenziell sind. Auch wenn sie sich teilweise nach außen richten, wie bei der Mittelbeschaffung, wird davon ausgegangen, dass der Fokus hauptsächlich nach innen auf die Organisation gerichtet ist, da sie in erster Linie von den Aktivitäten profitiert. 
Schließlich fasst das Feld der Öffentlichkeitsarbeit und Interessenvertretung die Tätigkeitsinhalte ,Informations- und Öffentlichkeitsarbeit', ,Interessenvertretung und Mitsprache sowie ,Vernetzungsarbeit' zusammen (Abbildung 11-1).
Diese Tätigkeitsinhalte können als Schnittstellenaufgaben gesehen werden, mit denen Verbindungen zwischen den Interessen und Belangen einer Organisation oder Gruppe und der Öffentlichkeit hergestellt werden.

\section{Abbildung 11-2: Hauptinhalte der freiwilligen Tätigkeit 2014}

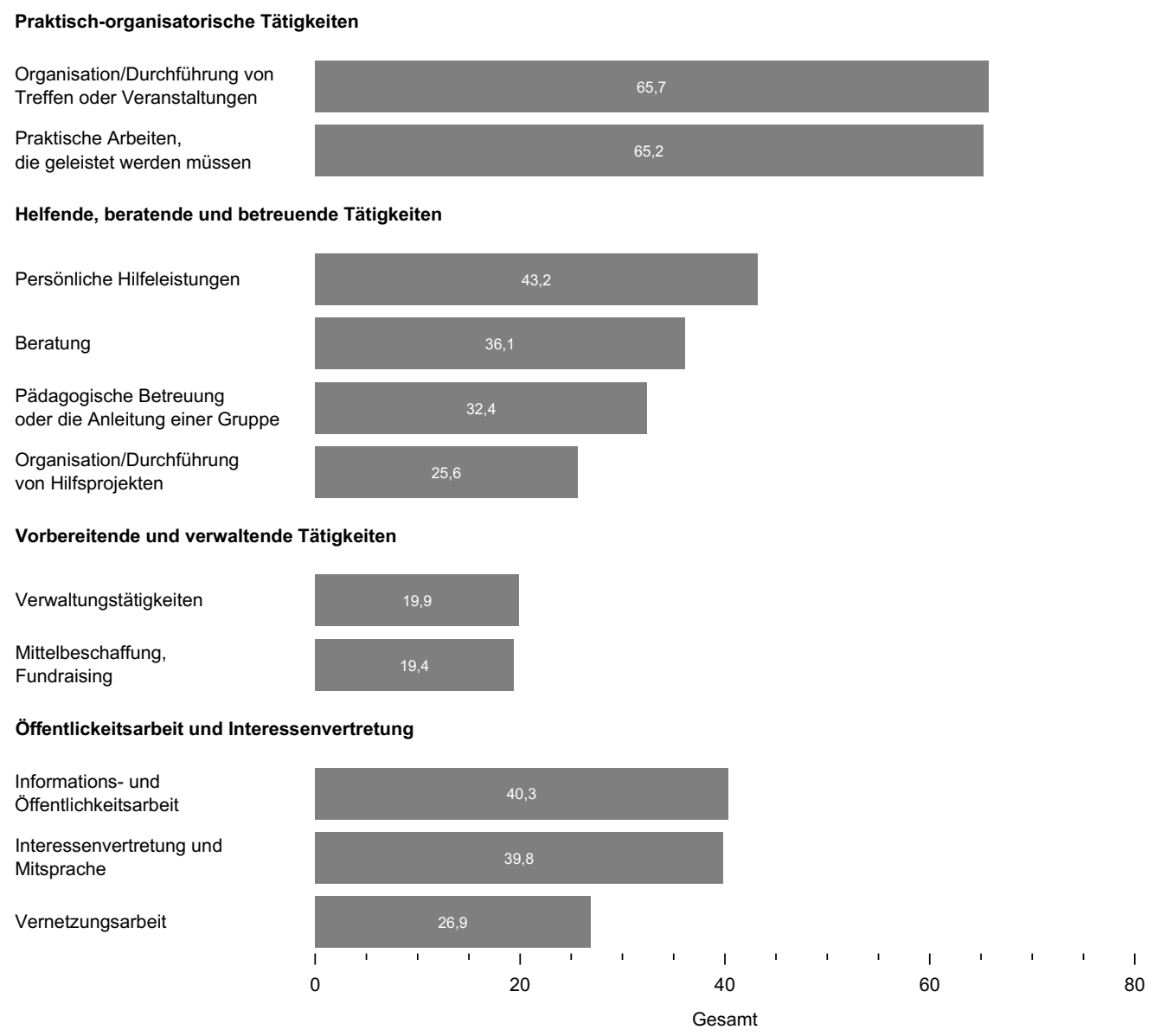

Quelle: FWS 2014, gewichtet, eigene Berechnungen (DZA). Basis: Alle Engagierten. Organisation/Durchführung von Treffen oder Veranstaltungen $(n=12.403)$, Praktische Arbeiten, die geleistet werden müssen $(n=12.390)$, Persönliche Hilfeleistungen ( $n=12.313)$, Beratung $(n=12.401)$, Pädagogische Betreuung oder die Anleitung einer Gruppe ( $n=12.401)$, Organisation/Durchführung von Hilfsprojekten ( $n=12.355)$, Verwaltungstätigkeiten $(n=12.404)$, Mittelbeschaffung, Fundraising ( $n=12.281)$, Informations- und Öffentlichkeitsarbeit $(n=12.387)$, Interessenvertretung und Mitsprache $(n=12.353)$, Vernetzungsarbeit $(n=12.313)$.

In Abbildung 11-2 wird gezeigt, welche nach Aussagen der Engagierten die Hauptinhalte ihrer freiwilligen Tätigkeiten sind. Auffällig ist, dass die praktisch-organisatorischen Tätigkeiten hierbei stark dominieren. Fast zwei Drittel aller freiwillig Engagierten berichten, dass die Haupt- 
inhalte ihrer Tätigkeit entweder die Organisation und Durchführung von Treffen und Veranstaltungen oder praktische Arbeiten, die geleistet werden müssen, umfassen. Helfende, beratende und betreuende Tätigkeiten werden ebenfalls häufig genannt, wobei hier die persönlichen Hilfeleistungen mit 43,2 Prozent und die Beratung mit 36,1 Prozent überwiegen. Nicht minder häufig genannte Tätigkeiten sind die Öffentlichkeitsarbeit und Interessenvertretung. Jeweils um die 40 Prozent aller freiwillig Engagierten sind in der Informations- und Öffentlichkeitsarbeit tätig oder geben Interessenvertretung und Mitsprache als Hauptinhalte ihrer Tätigkeit an. Am seltensten werden vorbereitende und verwaltende Tätigkeiten genannt.

In Abbildung 11-3 wird gezeigt, dass sich die Inhalte der Tätigkeiten teilweise zwischen Frauen und Männern unterscheiden. Auffällig ist dabei, dass Männer insbesondere den eher administrativen Tätigkeiten häufiger nachgehen als
Frauen. So geben Männer häufiger vorbereitende und verwaltende Tätigkeiten sowie Öffentlichkeitsarbeit und Interessenvertretung an als Frauen. Ebenso wird von Männern häufiger als von Frauen die Beratung als Haupttätigkeitsinhalt ihres Engagements benannt. Von Frauen werden hingegen persönliche Hilfeleistungen etwas häufiger genannt.

Es gibt allerdings auch eine Reihe von Tätigkeiten, die für beide Geschlechter ähnlich relevant sind: Dies zeigt sich beispielsweise bei dem Tätigkeitsfeld der praktisch-organisatorischen Tätigkeiten, aber auch bei der pädagogischen Betreuung oder Anleitung einer Gruppe sowie bei der Organisation/Durchführung von Hilfsprojekten. Bei diesen Tätigkeitsinhalten zeigen sich keine beziehungsweise nur sehr geringe geschlechtsspezifische Unterschiede. Darüber hinaus ist die Rangfolge der Tätigkeitsinhalte bei Frauen und Männern insgesamt sehr ähnlich (Abbildung 11-3). 


\section{Abbildung 11-3: Hauptinhalte der freiwilligen Tätigkeit 2014, nach Geschlecht}

\section{Praktisch-organisatorische Tätigkeiten}

Organisation/Durchführung von Treffen oder Veranstaltungen

Praktische Arbeiten,

die geleistet werden müssen

\begin{tabular}{|c|}
\hline 64,8 \\
\hline 66,6 \\
\hline 64,6 \\
\hline 65,8 \\
\hline
\end{tabular}

Helfende, beratende und betreuende Tätigkeiten

Persönliche Hilfeleistungen

\begin{tabular}{|c|}
\hline \multicolumn{1}{|c|}{45,1} \\
\hline 41,5 \\
\hline 32,4 \\
\hline 39,7 \\
\hline
\end{tabular}

Beratung

Pädagogische Betreuung oder die Anleitung einer Gruppe

Organisation/Durchführung von Hilfsprojekten

$\begin{array}{r}32,5 \\ \hline 32,3 \\ \hline\end{array}$

$\begin{array}{r}25,4 \\ 25,7 \\ \hline\end{array}$

Vorbereitende und verwaltende Tätigkeiten

Verwaltungstätigkeiten

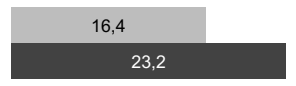

Mittelbeschaffung,

Fundraising

\begin{tabular}{|r|}
\hline 16,8 \\
\hline 21,9 \\
\hline
\end{tabular}

\section{Öffentlickeitsarbeit und Interessenvertretung}

Informations- und

Öffentlichkeitsarbeit

Interessenvertretung und Mitsprache

Vernetzungsarbeit

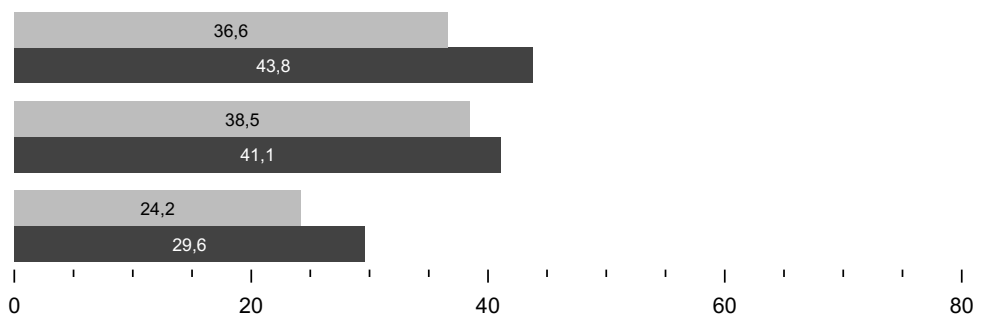

Prozent

Quelle: FWS 2014, gewichtet, eigene Berechnungen (DZA). Basis: Alle Engagierten. Organisation/Durchführung von Treffen oder Veranstaltungen $(n=12.403)$, Praktische Arbeiten, die geleistet werden müssen $(n=12.390)$, Persönliche Hilfeleistungen ( $n=12.313$ ), Beratung $(n=12.401)$, Pädagogische Betreuung oder die Anleitung einer Gruppe $(n=12.401)$, Organisation/Durchführung von Hilfsprojekten $(n=12.355)$, Verwaltungstätigkeiten $(n=12.404)$, Mittelbeschaffung, Fundraising ( $n=12.281)$, Informations- und Öffentlichkeitsarbeit $(n=12.387)$, Interessenvertretung und Mitsprache $(n=12.353)$, Vernetzungsarbeit $(n=12.313)$. 
Die Analysen zeigen zudem einige altersspezifische Unterschiede der Tätigkeitsinhalte, wobei hier kein einheitliches Muster innerhalb der vier Tätigkeitsfelder festzustellen ist (Abbildung 11-4). Auffällig ist, dass bei den vorbereitenden und verwaltenden Tätigkeiten Ältere dominieren. Auch die Beratung sowie die Informations- und
Öffentlichkeitsarbeit geben überdurchschnittlich mehr ältere Engagierte als Hauptinhalt ihres Engagements an. Jüngere Engagierte nennen hingegen häufiger als ältere Engagierte die pädagogische Betreuung oder Anleitung einer Gruppe sowie die Organisation/Durchführung von Treffen oder Veranstaltungen als Tätigkeitsinhalt. 


\section{Abbildung 11-4: Hauptinhalte der freiwilligen Tätigkeit 2014, nach Alter}

\section{Praktisch-organisatorische Tätigkeiten}

Organisation/Durchführung von Treffen oder Veranstaltungen

Praktische Arbeiten, die geleistet werden müssen

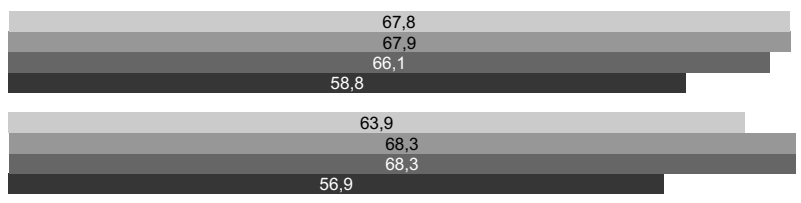

Helfende, beratende und betreuende Tätigkeiten

Persönliche Hilfeleistungen

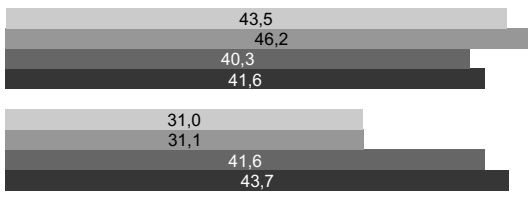

Pädagogische Betreuung oder die Anleitung einer Gruppe

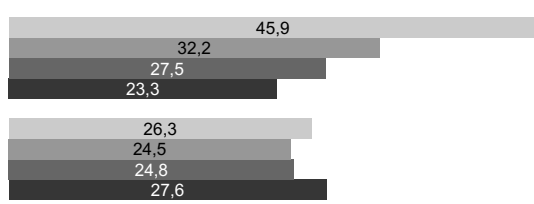

Organisation/Durchführung von Hilfsprojekten
Vorbereitende und verwaltende Tätigkeiten

Verwaltungstätigkeiten

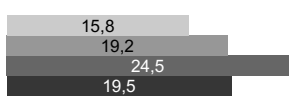

Mittelbeschaffung,

Fundraising

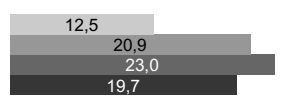

Öffentlickeitsarbeit und Interessenvertretung

Informations- und

Öffentlichkeitsarbeit

Interessenvertretung und Mitsprache

Vernetzungsarbeit

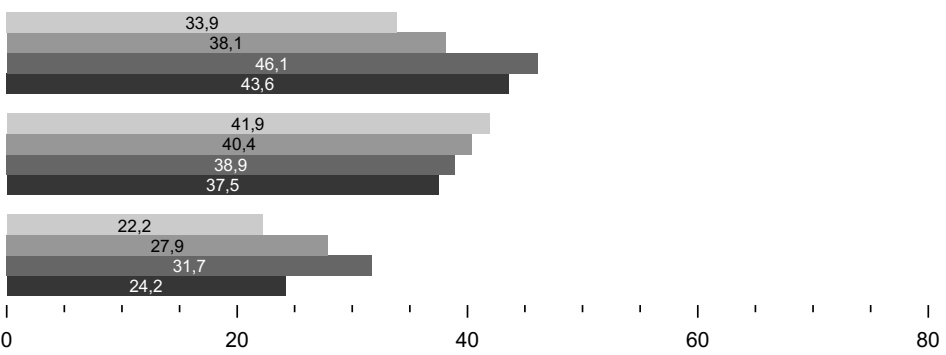

Prozent

Quelle: FWS 2014, gewichtet, eigene Berechnungen (DZA). Basis: Alle Engagierten. Organisation/Durchführung von Treffen oder Veranstaltungen $(n=12.403)$, Praktische Arbeiten, die geleistet werden müssen $(n=12.390)$, Persönliche Hilfeleistungen ( $n=12.313$ ), Beratung $(n=12.401)$, Pädagogische Betreuung oder die Anleitung einer Gruppe ( $n=12.401)$, Organisation/Durchführung von Hilfsprojekten $(n=12.355)$, Verwaltungstätigkeiten $(n=12.404)$, Mittelbeschaffung, Fundraising ( $n=12.281$ ), Informations- und Öffentlichkeitsarbeit $(n=12.387)$, Interessenvertretung und Mitsprache $(n=12.353)$, Vernetzungsarbeit $(n=12.313)$. 
Auch bei den bildungsspezifischen Unterschieden zeigt sich kein einheitliches Muster innerhalb beziehungsweise zwischen den vier Tätigkeitsfeldern (Abbildung 11-5). Engagierte mit hoher Bildung dominieren bei den vorbereitenden und verwaltenden Tätigkeiten und bei der Vernetzungsarbeit sowie der pädagogischen Betreuung oder Anleitung einer Gruppe. Engagierte mit niedriger oder mittlerer Bildung sind hingegen häufiger als Personen mit hoher Bildung bei den praktischen Arbeiten, die geleistet werden müssen sowie den persönlichen Hilfeleistungen, bei der Organisation/Durchführung von Hilfsprojekten und bei der Interessenvertretung und Mitsprache tätig. Keine signifikanten Bildungs- unterschiede zeigen sich bei der Organisation/ Durchführung von Treffen oder Veranstaltungen, und (mit Ausnahme der Schülerinnen und Schüler) bei der Informations- und Öffentlichkeitsarbeit sowie bei der Beratung.

Die Schülerinnen und Schüler nehmen eine besondere Stellung ein. Sie übernehmen im Vergleich zu den Engagierten, die bereits einen Schulabschluss haben, insbesondere häufiger eine pädagogische Betreuung oder die Anleitung einer Gruppe, jedoch signifikant seltener Tätigkeiten wie Vernetzungsarbeit, Verwaltungstätigkeiten, Beratung und Informations- und Öffentlichkeitsarbeit sowie Mittelbeschaffung/ Fundraising. 


\section{Abbildung 11-5: Hauptinhalte der freiwilligen Tätigkeit 2014, nach Bildung}

\section{Praktisch-organisatorische Tätigkeiten}

Organisation/Durchführung von Treffen oder Veranstaltungen

Praktische Arbeiten,

die geleistet werden müssen

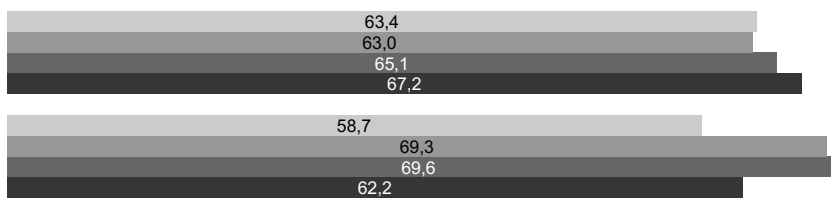

Helfende, beratende und betreuende Tätigkeiten

Persönliche Hilfeleistungen

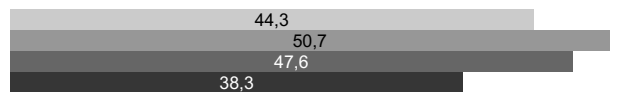

Beratung

Pädagogische Betreuung oder die Anleitung einer Gruppe

Organisation/Durchführung von Hilfsprojekten

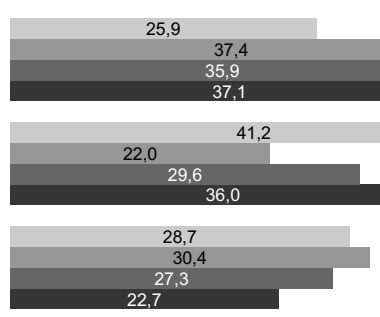

Vorbereitende und verwaltende Tätigkeiten

Verwaltungstätigkeiten

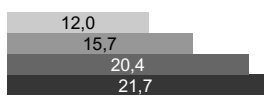

Mittelbeschaffung,

Fundraising

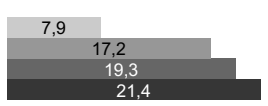

Öffentlickeitsarbeit und Interessenvertretung

Informations- und

Öffentlichkeitsarbeit

Interessenvertretung und Mitsprache

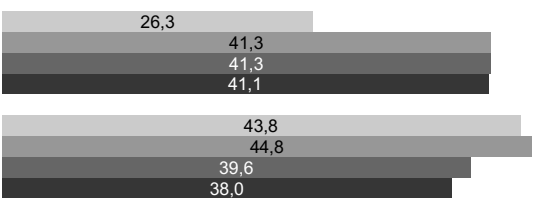

Vernetzungsarbeit

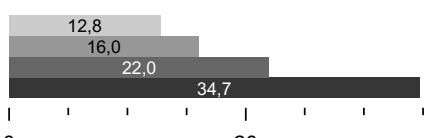

Prozent

Quelle: FWS 2014, gewichtet, eigene Berechnungen (DZA). Basis: Alle Engagierten. Organisation/Durchführung von Treffen oder Veranstaltungen $(n=12.400)$, Praktische Arbeiten, die geleistet werden müssen $(n=12.387)$, Persönliche Hilfeleistungen ( $n=12.310)$, Beratung $(n=12.398)$, Pädagogische Betreuung oder die Anleitung einer Gruppe $(n=12.398)$, Organisation/Durchführung von Hilfsprojekten $(n=12.352)$, Verwaltungstätigkeiten $(n=12.401)$, Mittelbeschaffung, Fundraising ( $n=12.278)$, Informations- und Öffentlichkeitsarbeit $(n=12.384)$, Interessenvertretung und Mitsprache $(n=12.350)$, Vernetzungsarbeit $(n=12.310)$. 
Im Zeitvergleich wird ersichtlich, dass engagierte Personen zunehmend mehr Tätigkeitsinhalte angeben (Abbildung 11-6). In fast allen Kategorien ist über die Zeit eine kontinuierliche Zunahme von Angaben zu verzeichnen. Die Rangfolge der Tätigkeitsinhalte ist jedoch trotz dieser Zunahme seit 1999 mit wenigen Ausnahmen stabil geblieben. Als die beiden häufigsten Inhalte werden durchgängig praktisch-organisatorische Tätigkeiten, also die Organisation oder Durchführung von Treffen und Veranstaltungen sowie praktische Arbeiten, die geleistet werden müssen, genannt. Besonders große Zuwächse im Vergleich zu 2009 sind bei den praktischen Arbeiten, bei der Beratung sowie bei der Vernetzungsarbeit zu beobachten.

Die Zunahme der Angaben über fast alle Tätigkeitsinhalte hinweg gibt einen Hinweis darauf, dass Engagierte immer mehr unterschiedliche Aufgaben haben und es eine größere Formenvielfalt der Tätigkeiten gibt. Vor dem Hintergrund, dass sich das Zeitbudget für die freiwillige Tä- tigkeit im Zeitvergleich nicht erhöht hat (siehe Kapitel 12), ist davon auszugehen, dass sich die Tätigkeitsdichte erhöht hat, also mehr inhaltliche Aufgaben in der gleichen Zeit ausgeübt werden.

Betrachtet man die Entwicklung von Geschlechterunterschieden in den Tätigkeitsinhalten im Zeitvergleich (Tabelle 11-1 im Online-Anhang), so fällt auf, dass hier bei den meisten Inhalten zwar keine Annäherung stattgefunden hat, sich bestehende Unterschiede aber auch nicht verstärkt haben. Bei Tätigkeitsinhalten mit einer unterschiedlichen Verteilung zwischen den Geschlechtern, wie der Informations- und Öffentlichkeitsarbeit oder den Verwaltungstätigkeiten, sind die Geschlechterunterschiede über die Zeit betrachtet vergleichsweise konstant geblieben. Bei Tätigkeitsinhalten, die von Frauen und Männern nahezu gleichermaßen häufig ausgeübt werden, wie der Betreuung oder Anleitung einer Gruppe, gab es auch in der Vergangenheit keine oder nur sehr geringe Geschlechterunterschiede. 


\section{Abbildung 11-6: Hauptinhalte der freiwilligen Tätigkeit im Zeitvergleich}

\section{Praktisch-organisatorische Tätigkeiten}

Organisation/Durchführung von

Treffen oder Veranstaltungen

Praktische Arbeiten,

die geleistet werden müssen

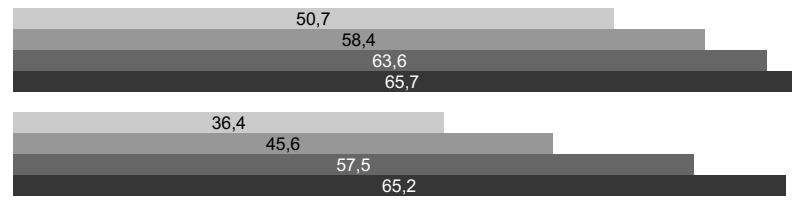

Helfende, beratende und betreuende Tätigkeiten

Persönliche Hilfeleistungen

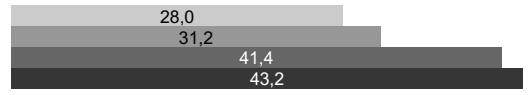

Beratung

Pädagogische Betreuung oder die Anleitung einer Gruppe

Organisation/Durchführung von Hilfeprojekten
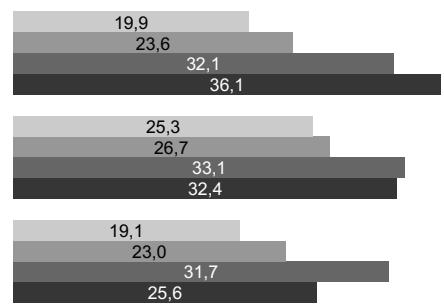

Vorbereitende und verwaltende Tätigkeiten

Verwaltungstätigkeiten

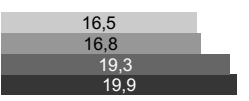

Mittelbeschaffung,

Fundraising

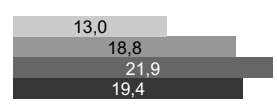

\section{Öffentlickeitsarbeit und Interessenvertretung}

Informations- und

Öffentlichkeitsarbeit

Interessenvertretung und

Mitsprache

Vernetzungsarbeit
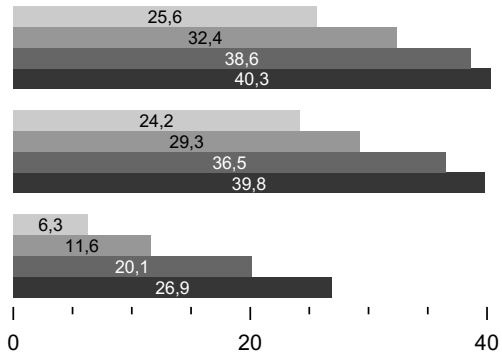

40

Prozent

Quelle: FWS, gewichtet, eigene Berechnungen (DZA). Basis: Alle Engagierten. FWS 1999 ( $n=4.678)$, FWS 2004 ( $n=5.126)$, FWS 2009 ( $n=6.990)$, FWS 2014: Organisation/Durchführung von Treffen oder Veranstaltungen $(n=12.403)$, Praktische Arbeiten, die geleistet werden müssen $(n=12.390)$, Persönliche Hilfeleistungen $(n=12.313)$, Beratung $(n=12.401)$, Pädagogische Betreuung oder die Anleitung einer Gruppe $(n=12.401)$, Organisation/Durchführung von Hilfsprojekten ( $n=12.355)$, Verwaltungstätigkeiten ( $n=12.404)$, Mittelbeschaffung, Fundraising ( $n=12.281)$, Informations- und Öffentlichkeitsarbeit $(n=12.387)$, Interessenvertretung und Mitsprache $(n=12.353)$, Vernetzungsarbeit $(n=12.313)$. 


\subsection{Leitungs- und Vorstandstätigkeiten im freiwilligen Engagement}

Insgesamt 27,5 Prozent aller Engagierten üben im Rahmen ihrer freiwilligen Tätigkeit eine Leitungs- oder Vorstandstätigkeit aus (Abbildung 11-7a). Dabei haben Männer mit 33,0 Prozent deutlich häufiger eine Leitungs- oder Vorstandsfunktion als Frauen mit 21,7 Prozent. Der Anteil der Engagierten, die eine leitende Tätigkeit ausüben, ist in der Altersgruppe der 50- bis 64-Jährigen am höchsten, gefolgt von Personen ab 65 Jahren. Etwas seltener sind Leitungsfunktionen im Engagement bei 30- bis 49-Jährigen und am seltensten in der jüngsten hier ausgewiesenen Altersgruppe. Dabei scheint insbesondere bei den Männern das Alter für die Ausübung einer Leitungs- oder Vorstandstätigkeit eine Rolle zu spielen, denn hier sind die Unterschiede zwischen den Altersgruppen wesentlich deutlicher zu erkennen als bei den Frauen: Während bei den 50- bis 64-jährigen Männern 40,3 Prozent und bei den 65-jährigen und älteren Männern 39,8 Prozent der Engagierten eine Leitungs- oder
Vorstandsfunktion ausüben, sind es bei den 14bis 29-jährigen Männern lediglich 22,2 Prozent. Bei den Frauen gibt es vergleichsweise geringe Unterschiede zwischen den Altersgruppen ( $\mathrm{Ta}$ belle 11-8 im Online-Anhang). In der jüngsten Altersgruppe, bei den 14- bis 29-Jährigen, ist der Anteil der engagierten Frauen und Männer mit Leitungs- oder Vorstandsfunktion allerdings ähnlich hoch. Dies ist möglicherweise ein Hinweis darauf, dass die Geschlechterdifferenzen bei der Übernahme von Leitungs- oder Vorstandsfunktionen in Zukunft geringer ausfallen könnten.

Leitungs- und Vorstandstätigkeiten werden nicht nur von Höhergebildeten ausgeübt (29,3 Prozent), sondern annähernd ebenso häufig auch von Personen mit niedriger (28,0 Prozent) oder mittlerer Bildung (26,7 Prozent; Abbildung 11-7b). Lediglich Schülerinnen und Schüler üben deutlich seltener eine Leitungs- oder Vorstandstätigkeit im Engagement aus. 
Abbildung 11-7: Anteile der Personen mit Leitungs- und Vorstandstätigkeiten im freiwilligen Engagement $\mathbf{2 0 1 4}$, a) gesamt, nach Geschlecht und nach Alter und b) nach Bildung

a) gesamt, nach Geschlecht und nach Alter

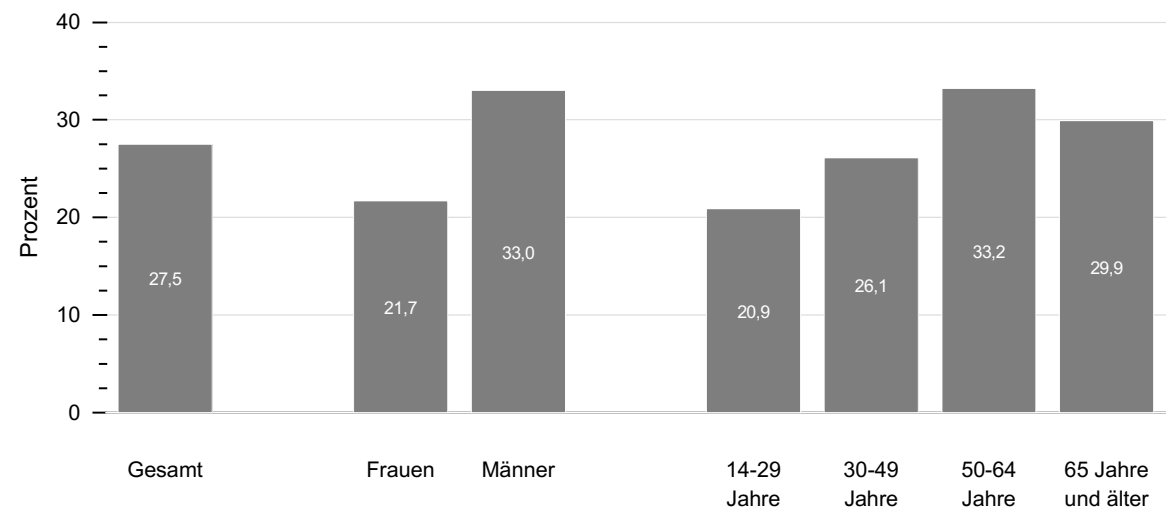

b) nach Bildung

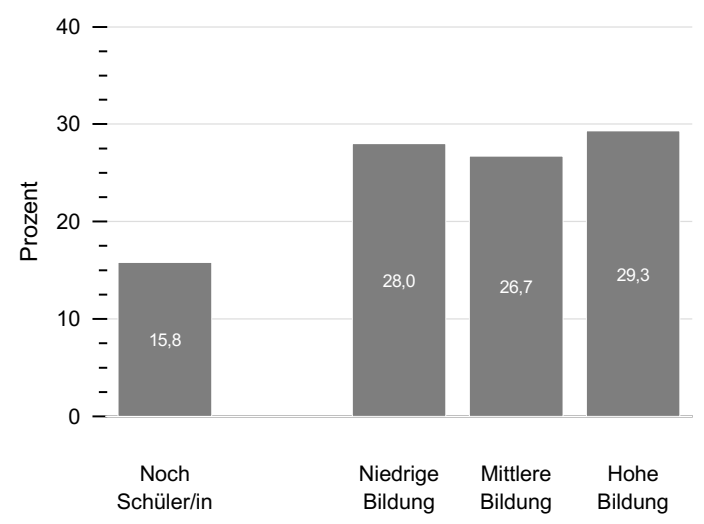

Quelle: FWS 2014, gewichtet, eigene Berechnungen (DZA). Basis: Alle Engagierten. Gesamt, nach Geschlecht und nach Alter ( $n=12.409)$, nach Bildung $(n=12.406)$. 
Der in Abbildung 11-8 zu beobachtende Trend, der zeigt, dass seit 1999 immer weniger Engagierte eine Leitungs- oder Vorstandstätigkeit haben, basiert auf der Betrachtung aller Engagierten. Hiernach sank der Anteil der Engagierten mit Leitungs- und Vorstandsfunktion von 38,2 Prozent im Jahr 1999 um gut zehn Prozentpunkte auf 27,5 Prozent im Jahr 2014. Dabei ist der Rückgang für beide Geschlechter nahezu gleichermaßen stark ausgeprägt. Er findet allerdings auf unterschiedlichem Niveau statt, da der Anteil der engagierten Frauen mit Leitungs- oder Vorstandsfunktion in allen Erhebungsjahren deutlich niedriger ist als der der engagierten Männer (Tabelle 11-8 im Online-Anhang).

Werden diese Befunde jedoch ins Verhältnis zur gesamten Wohnbevölkerung im Alter ab 14 Jahren gesetzt, zeigt sich, dass der relative
Rückgang der Leitungs- und Vorstandstätigkeit innerhalb der Gruppe der Engagierten nicht mit einem Rückgang der absoluten Zahl der Leitungs- und Vorstandstätigen einhergeht. Im Jahr 1999 übten 12,9 Prozent der Gesamtbevölkerung in Deutschland ein freiwilliges Engagement mit einer Leitungs- oder Vorstandstätigkeit aus, 2004 waren es 12,5 Prozent und 2009 noch 11,6 Prozent, im Jahr 2014 beträgt dieser Anteil 12,0 Prozent, wobei die Schwankungen über die Zeit insgesamt statistisch nicht signifikant sind. Dies bedeutet, dass der zu beobachtende relative Rückgang von Engagierten mit Leitungs- und Vorstandstätigkeit an allen Engagierten darauf zurückzuführen ist, dass die Zahl der Engagierten ohne Leitungs- oder Vorstandstätigkeit stark zugenommen hat.

\section{Abbildung 11-8: Anteile der Personen mit Leitungs- und Vorstandstätigkeiten im Engagement im Zeitvergleich}

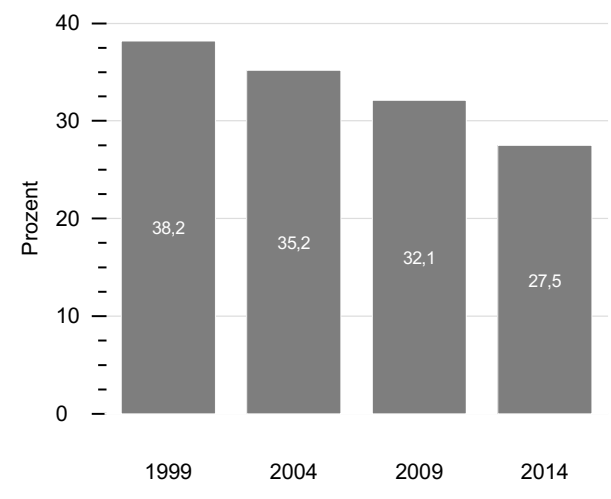

Quelle: FWS, gewichtet, eigene Berechnungen (DZA). Basis: Alle Engagierten. FWS 1999 ( $n=4.672$ ), FWS 2004 ( $n=5.124)$, FWS 2009 ( $n=6.986)$, FWS 2014 ( $n=12.409)$,

Das Verhältnis zwischen den unterschiedlichen Altersgruppen hat sich hinsichtlich der Leitungsund Vorstandsfunktionen im Zeitvergleich kaum geändert: In allen Untersuchungsjahren haben die 50- bis 64-Jährigen am häufigsten und die jüngste Altersgruppe der 14- bis 29-Jährigen am seltensten eine Leitungs- oder Vorstandsfunktion. Allerdings nimmt der Anteil der 65-Jährigen und Älteren mit Leitungs- oder Vorstandsfunk- tion tendenziell etwas zu (Tabelle 11-8 im Online-Anhang).

Das Verhältnis zwischen den unterschiedlichen Altersgruppen hat sich hinsichtlich der Leitungs- und Vorstandsfunktionen im Zeitvergleich kaum geändert: In allen Untersuchungsjahren haben die 50 - bis 64-Jährigen am häufigsten und die jüngste Altersgruppe der 14- bis 29-Jährigen am seltensten eine Leitungs- oder Vorstandsfunktion. Allerdings nimmt der Anteil 
der 65-Jährigen und Älteren mit Leitungs- oder Vorstandsfunktion tendenziell etwas zu (Tabelle 11-8 im Online-Anhang).

Im Zeitvergleich haben sich, in Bezug auf die Leitungs- und Vorstandsfunktionen im Engagement, die Bildungsgruppen deutlich angenähert. Während 2014 kaum Bildungsunterschiede festzustellen sind (Abbildung 11-7b), standen beispielsweise 2009 noch 28,5 Prozent Engagierte mit Leitungs- oder Vorstandstätigkeit bei den Niedriggebildeten 35,6 Prozent bei den Höhergebildeten gegenüber (Tabelle 11-8 im Online-An- hang). Dabei haben sich die Anteile der Engagierten mit Leitungsfunktion bei den Personen mit niedriger Bildung am wenigsten verringert. Den deutlichsten anteiligen Rückgang weisen im Zeitvergleich die Höhergebildeten auf. Das ist jedoch nicht darauf zurückzuführen, dass sich weniger hochgebildete Personen mit einer Leitungs- oder Vorstandsfunktionen engagieren, sondern dass es mehr Höhergebildete gibt, die eine freiwillige Tätigkeit ohne Leitungsfunktion ausüben.

\section{Abbildung 11-9: Anteile der Personen mit Leitungs- und Vorstandstätigkeiten im Engagement nach gesellschaftlichem Bereich 2014, gesamt und nach Geschlecht}

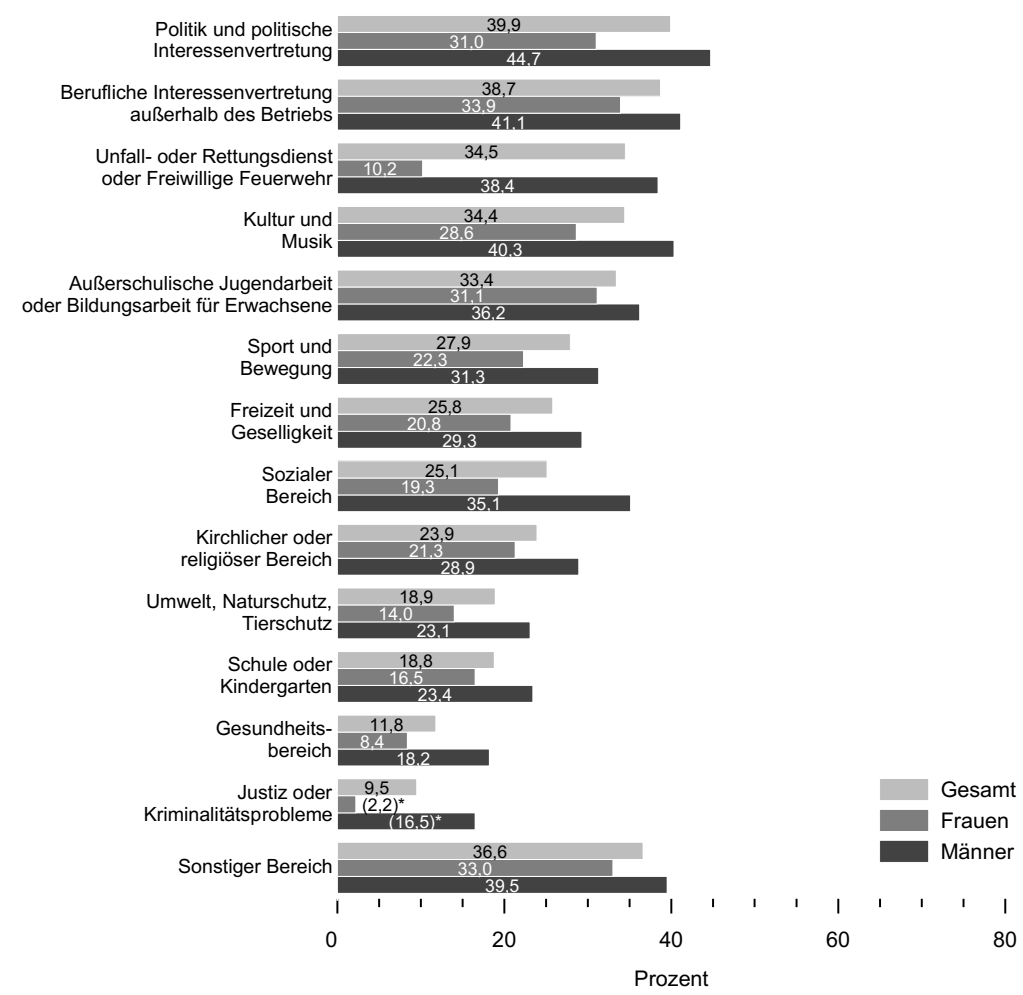

Quelle: FWS 2014, gewichtet, eigene Berechnungen (DZA). Basis: Alle Engagierten ( $n=12.320$ ).

Hinweis: * Für den Bereich ,Justiz oder Kriminalitätsprobleme‘ werden die Prozente der Männer und Frauen mit Leitungs- oder Vorstandsfunktion in Klammern ausgegeben, da die Prozentuierungsbasis $n<50$ ist.

Die Anteile der Engagierten mit Leitungs- und Vorstandsfunktion unterscheiden sich darüber hinaus deutlich zwischen den Bereichen. Während in einigen Bereichen wie Politik und politi- 
scher Interessenvertretung sowie der beruflichen Interessenvertretung und im Bereich Unfalloder Rettungsdienst mehr als ein Drittel der Engagierten eine Vorstands- oder Leitungsfunktion hat, ist es im Bereich Justiz und Kriminalitätsprobleme oder im Gesundheitsbereich nur etwa ein Zehntel der Engagierten (Abbildung 11-9). Diese Unterschiede spiegeln auch die in den jeweiligen Bereichen dominierenden Organisationsformen wider. Parteien, Verbände und Gewerkschaften stellen eher institutionalisierte Organisationen dar, die eine gesamtgesellschaftliche und politische Ausrichtung haben und in denen häufiger hierarchische Strukturen zu finden sind, die infolge auch häufiger mit Leitungs- und Vorstandsfunktionen verbunden sind.

Je nach gesellschaftlichem Bereich des Engagements zeigen sich hinsichtlich der Leitungsund Vorstandsfunktionen unterschiedlich stark ausgeprägte Geschlechterdifferenzen (Abbil- dung 11-9). Bezogen auf den jeweiligen Anteil engagierter Frauen und Männer üben in allen Engagementbereichen - unabhängig davon, ob es sich um einen Bereich handelt, in dem sich insgesamt mehr Frauen als Männer engagieren oder umgekehrt - Frauen seltener eine Leitungs- oder Vorstandstätigkeit aus als Männer. Besonders ausgeprägte Geschlechterdifferenzen hinsichtlich der Leitungs- und Vorstandstätigkeiten finden sich im sozialen Bereich (Frauen: 19,3 Prozent, Männer: 35,1 Prozent), im Bereich der Unfall- und Rettungsdienste und der Freiwilligen Feuerwehr (Frauen: 10,2 Prozent, Männer: 38,4 Prozent) sowie im Bereich Justiz oder Kriminalität (Frauen: 2,2 Prozent, Männer: 16,5 Prozent). Die Entwicklung der Leitungs- und Vorstandsfunktionen in den letzten fünfzehn Jahren innerhalb der Bereiche ist in den jeweiligen Bereichsprofilen im Anhang von Kapitel 3 abgebildet. 


\subsection{Internetnutzung im freiwilligen Engagement}

Das Internet hat in den letzten beiden Jahrzehnten deutlich an Bedeutung gewonnen und durchdringt praktisch alle Gesellschaftsbereiche. Auch für das freiwillige Engagement spielt das Internet eine nicht unbedeutende Rolle: Eine Mehrheit der Engagierten (58,4 Prozent) nutzt das Internet für ihre freiwillige Tätigkeit, Frauen (52,9 Pro- zent) dabei etwas seltener als Männer (63,7 Prozent; Abbildung 11-10a). Am häufigsten nutzen Personen in den mittleren Altersgruppen (30bis 49-Jährige: 64,0 Prozent; 40- bis 64-Jährige: 64,1 Prozent) das Internet für ihr freiwilliges Engagement, bei den Jüngeren und bei den Älteren ist die Nutzung des Internets im Engagement

Abbildung 11-10: Anteile der Personen, die das Internet für ihre freiwillige Tätigkeit nutzen, 2014, a) gesamt, nach Geschlecht und nach Alter sowie b) nach Bildung

a) gesamt, nach Geschlecht und nach Alter

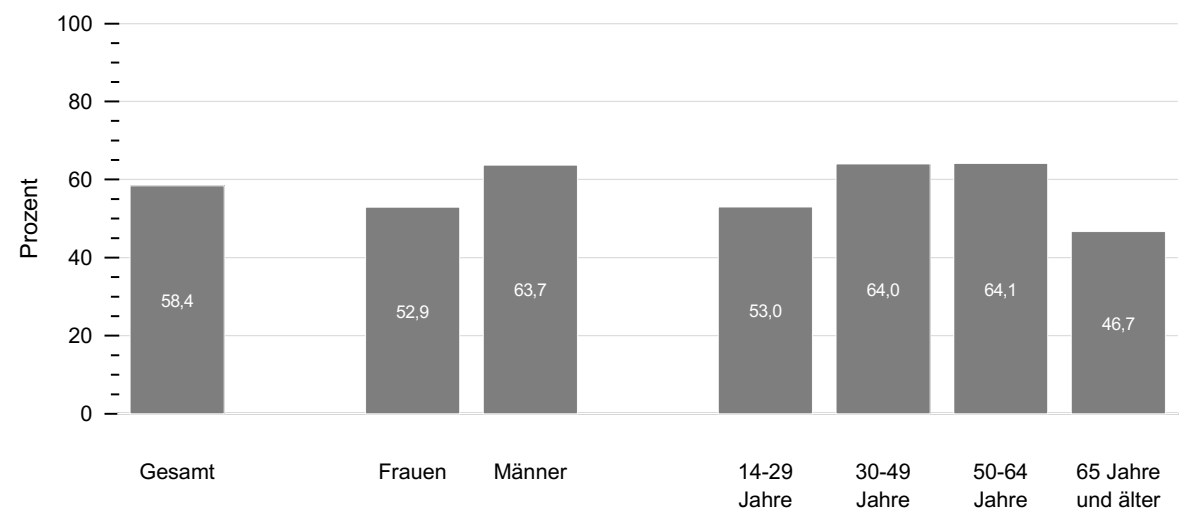

b) nach Bildung

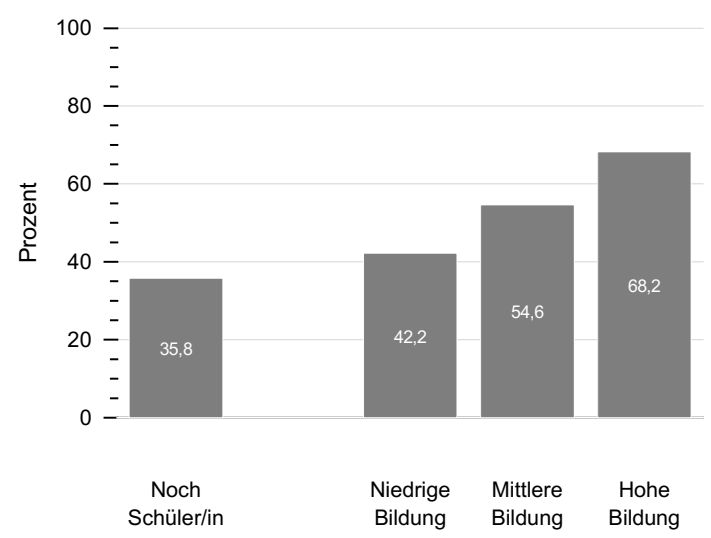

Quelle: FWS 2014, gewichtet, eigene Berechnungen (DZA). Basis: Alle Engagierten $(n=12.421)$. 
dagegen etwas weniger verbreitet (14- bis 29-Jährige: 53,0 Prozent; 65-Jährige und Ältere: 46,7 Prozent). Dies ist vor dem Hintergrund der bereits berichteten Unterschiede bei den Inhalten des Engagements zu sehen. So liegt ein Tätigkeitsschwerpunkt der jüngsten Altersgruppe auf der pädagogischen Anleitung oder Betreuung einer Gruppe. Hierbei wird das Internet vermutlich deutlich seltener genutzt als beispielsweise bei der Informations- und Öffentlichkeitsarbeit oder bei Vernetzungstätigkeiten, die häufiger von Personen im mittleren Lebensalter ausgeübt werden.

Bei der Internetnutzung gibt es außerdem einen starken Bildungsgradienten (Abbildung 11-10b). Personen mit höherer Bildung nutzen das Internet bei ihrer freiwilligen Tätigkeit deutlich häufiger als Personen mit mittlerer Bildung, bei denen die Internetnutzung wiederum verbreiteter ist als bei Personen mit niedriger Bildung. Schülerinnen und Schüler nutzen das Internet am seltensten für ihre freiwillige Tätigkeit. Dieser Befund spiegelt die bereits berichteten Altersunterschiede wider. Männer nutzen das Internet in allen Altersgruppen häufiger bei ihrer freiwilligen Tätigkeit als Frauen (Tabelle 11-10 im Online-Anhang). Dieser Unterschied ist bei den älteren Engagierten besonders stark ausgeprägt. So nutzen im Alter von 65 und mehr Jahren 59,4 Prozent der engagierten Männer, aber nur 33,3 Prozent der engagierten Frauen das Internet für ihre Tätigkeit. Im Vergleich zum Jahr 2009 haben sich die Anteile der Internetnutzung im Engagement insgesamt nur geringfügig verändert (Tabelle 11-10 im Online-Anhang).

Von insgesamt 18,5 Prozent aller Engagierten wird das Internet auch interaktiv genutzt, also zum Beispiel durch das Betreiben von Blogs oder über soziale Netzwerke (Abbildung 11-11a). Auch hier ist der Anteil bei den Frauen deutlich niedriger (15,2 Prozent) als bei den Männern (21,8 Prozent). Bei der interaktiven Nutzung des
Internets dominieren insbesondere die Jüngeren: 28,9 Prozent der 14- bis 29-jährigen Engagierten nutzen das Internet interaktiv, aber nur 8,0 Prozent der Personen ab 65 Jahren. Setzt man die interaktive und die allgemeine Internetnutzung ins Verhältnis, so fällt auf, dass bei den Jüngeren zwar das Internet insgesamt vergleichsweise selten im Rahmen des freiwilligen Engagements genutzt wird. Wenn Personen zwischen 14 und 29 Jahren jedoch das Internet für ihre Tätigkeit nutzen, dann häufig auch interaktiv, etwa indem sie Inhalte in sozialen Netzwerken teilen oder selbst Blogs betreiben.

Ähnlich wie bei der Internetnutzung insgesamt, sind auch hier deutliche Unterschiede nach Bildungsgruppen festzustellen. Mit dem Bildungsniveau steigt auch der Anteil derjenigen, die in ihrer freiwilligen Tätigkeit interaktiv das Internet nutzen. Besonders hohe Raten sind für die Schülerinnen und Schüler festzustellen, dies entspricht den berichteten Altersunterschieden. Auch bei der interaktiven Internetnutzung sind in allen Altersgruppen die Raten der Frauen niedriger als die der Männer.

Nur bei einem sehr geringen Teil der Engagierten findet das Engagement ausschließlich (0,6 Prozent) oder überwiegend (2,1 Prozent) im Internet statt (Tabelle 11-12 im Online-Anhang). In der jüngsten Altersgruppe wird das Engagement etwas häufiger als in anderen Altersgruppen überwiegend über das Internet ausgeübt (3,3 Prozent); ausschließlich über das Internet engagieren sich aber auch in dieser Altersgruppe nur 0,7 Prozent der Engagierten. Die Internetnutzung stellt in der Regel also eine Erweiterung der freiwilligen Tätigkeit dar, ersetzt andere Tätigkeitsformen aber nur im Ausnahmefall. Online-Volunteering, im Sinne einer ausschließlich oder überwiegend über das Internet ausgeübten Tätigkeit, ist insofern als Phänomen zwar feststellbar, jedoch (noch) keine weit verbreitete Form des Engagements. 
Abbildung 11-11: Anteile der Personen mit interaktiver Nutzung des Internets für ihre freiwillige Tätigkeit 2014, a) gesamt, nach Geschlecht und nach Alter sowie b) nach Bildung

a) gesamt, nach Geschlecht und nach Alter

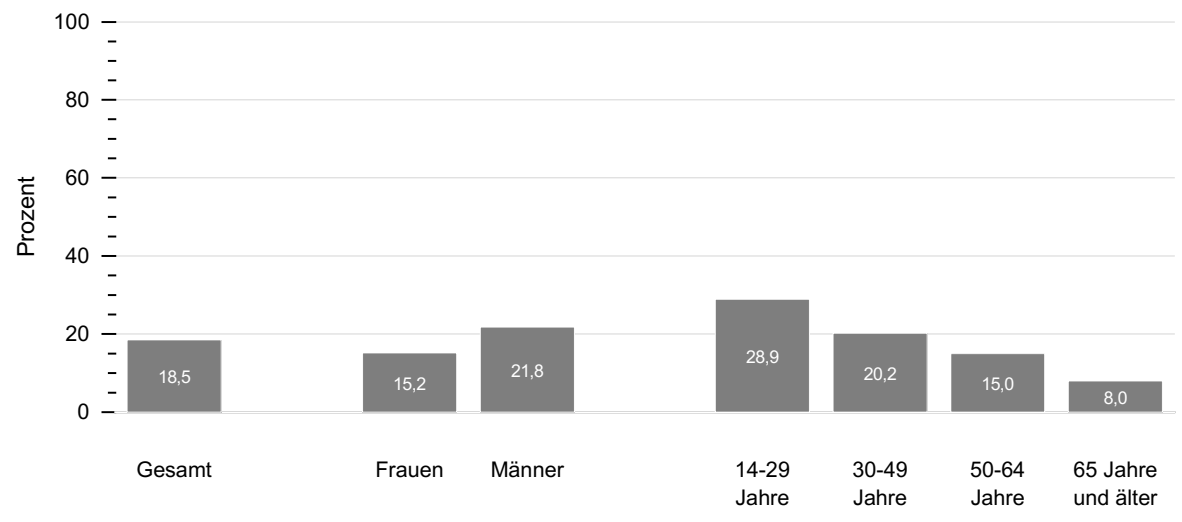

b) nach Bildung

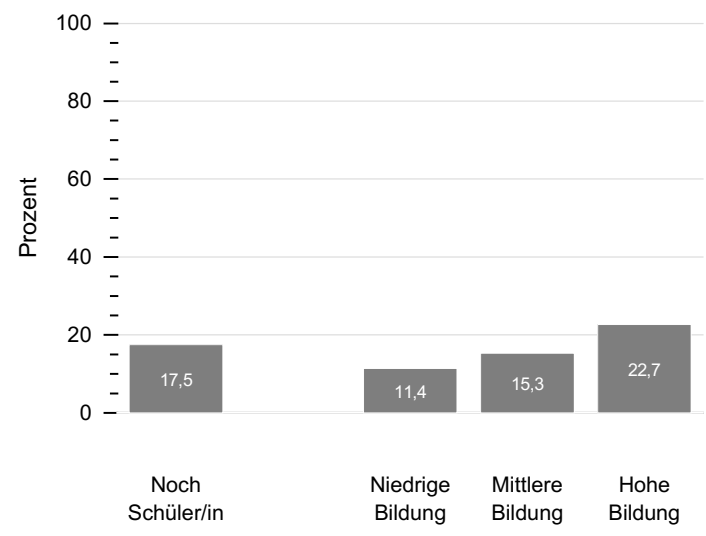

Quelle: FWS 2014, gewichtet, eigene Berechnungen (DZA). Basis: Alle Engagierten $(n=12.410)$. 


\subsection{Zielgruppen und Zielregionen des freiwilligen Engagements}

Freiwillige Tätigkeiten können sich an unterschiedliche Personengruppen richten und diesen zugutekommen. In Abbildung 11-12 sind die Zielgruppen der freiwilligen Tätigkeit dargestellt. Dabei können sich Engagierte für mehr als eine Zielgruppe einsetzen; in der Erhebung wurde dies durch die Möglichkeit der Mehrfachnennung berücksichtigt. Freiwilliges Engagement kann sich also beispielsweise sowohl auf Kinder und Jugendliche als auch auf ältere Menschen richten. Darüber hinaus kann eine Zielgruppe mehrere Eigenschaften aufweisen: Eine freiwillige Tätigkeit kann sich also beispielsweise an Menschen richten, die sowohl finanziell oder sozial schlechter gestellt sind als auch einen Migrationshintergrund haben.

Als häufigste Zielgruppe wird die der Kinder und Jugendlichen genannt. Knapp die Hälfte aller Engagierten bezieht sich mit ihrer freiwilligen Tätigkeit auf diese Gruppe (Abbildung 11-12).
Häufige Adressatinnen und Adressaten des freiwilligen Engagements sind darüber hinaus $\mathrm{Fa}$ milien sowie ältere Menschen, wobei sich Frauen jeweils etwas häufiger für diese Personengruppen engagieren als Männer. Bei jeweils knapp 15 Prozent der Engagierten richtet sich das Engagement auf hilfe- oder pflegebedürftige beziehungsweise auf finanziell oder sozial schlechter gestellte Menschen. Auch diese Zielgruppen werden eher von Frauen genannt. Gut elf Prozent der Engagierten richten sich mit ihrer Tätigkeit an Menschen mit Migrationshintergrund. Dies können ganz unterschiedliche Zuwanderungsgruppen sein, also beispielsweise aktuell nach Deutschland kommende Flüchtlinge, aber auch Menschen, die seit vielen Jahren in Deutschland leben oder hier geboren wurden, deren Eltern aber eingewandert sind. Dabei engagieren sich Frauen etwas häufiger für Menschen mit Migrationshintergrund als Männer. 


\section{Abbildung 11-12: Zielgruppen der freiwilligen Tätigkeit 2014, gesamt und nach Geschlecht}

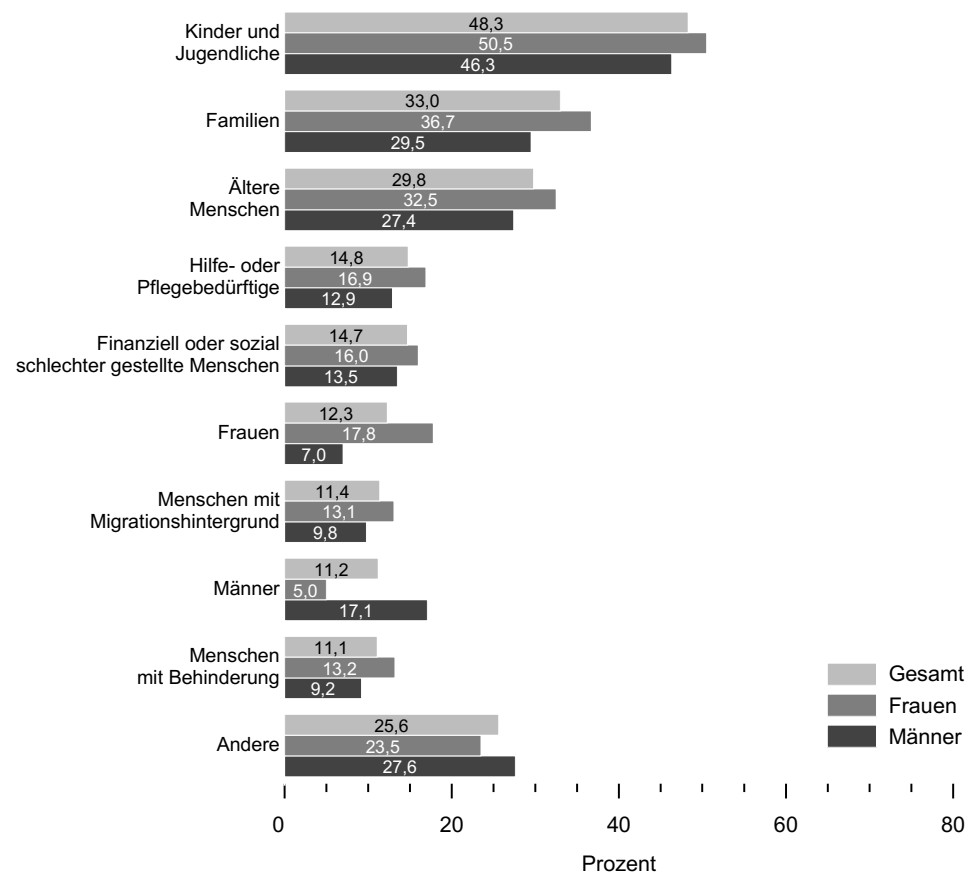

Quelle: FWS 2014, gewichtet, eigene Berechnungen (DZA). Basis: Alle Engagierten. Kinder und Jugendliche $(n=12.401)$, Familien ( $n=12.377)$, Ältere Menschen $(n=12.380)$, Hilfe- oder Pflegebedürftige $(n=12.394)$, Finanziell oder sozial schlechter gestellte Menschen $(n=12,276)$, Frauen $(n=12.394)$, Menschen mit Migrationshintergrund $(n=12.369)$, Männer ( $n=12.388)$, Menschen mit Behinderungen $(n=12.397)$, Andere $(n=12.340)$. 
Zu erwartende, deutliche Unterschiede zwischen den Geschlechtern finden sich hinsichtlich der Zielgruppe der Frauen (mehr Frauen als Männer engagieren sich für Frauen) und der Zielgruppe der Männer (mehr Männer als Frauen engagieren sich für Männer). Ein gutes Viertel der Engagierten gibt an, sich für eine andere, nicht näher definierte, Zielgruppe zu engagieren. Hier könnte man vermuten, dass sich diese Personen nicht explizit für eine oder mehrere andere Personengruppen engagieren, sondern dass sie sich entweder für Menschen im Allgemeinen oder aber für eine Idee oder eine Sache einsetzen.

Bei einer Differenzierung nach Alter zeigt sich, dass insbesondere die jüngere Generation bei ihrem Engagement Kinder und Jugendliche als Zielgruppe nennt. Bei den 14- bis 29-Jährigen sind das immerhin 68,6 Prozent und bei den 30- bis 49-Jährigen 57,3 Prozent. Bei den 65-Jährigen und Älteren beträgt der entsprechende Anteil lediglich 24,5 Prozent (Abbildung 11-13). Das freiwillige Engagement, welches sich an ältere Menschen, Menschen mit Behinderungen sowie an finanziell oder sozial schlechter gestellte Menschen richtet, wird hingegen häufiger von Älteren als von Jüngeren geleistet. Auffällig hierbei ist, dass bei allen drei genannten Zielgruppen signifikant häufiger Frauen ab 65 Jahren als Männer in diesem Alter engagiert sind (siehe Tabelle 11-2 im Online-Anhang).

\section{Abbildung 11-13: Zielgruppen der freiwilligen Tätigkeit 2014, nach Alter}

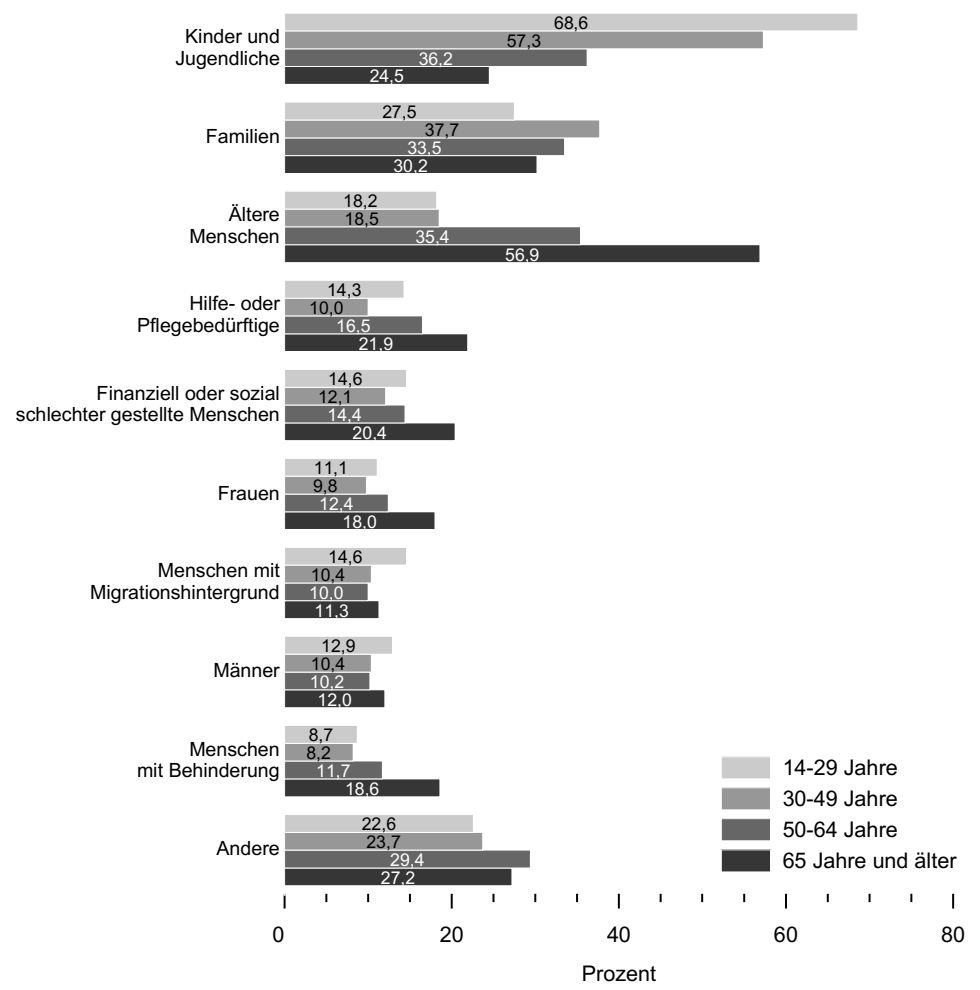

Quelle: FWS 2014, gewichtet, eigene Berechnungen (DZA). Basis: Alle Engagierten. Kinder und Jugendliche $(n=12.401)$, Familien ( $n=12.377)$, Ältere Menschen $(n=12.380)$, Hilfe- oder Pflegebedürftige $(n=12.394)$, Finanziell oder sozial schlechter gestellte Menschen $(n=12.276)$. Frauen $(n=12.394)$, Menschen mit Migrationshintergrund $(n=12.369)$, Männer ( $n=12.388)$, Menschen mit Behinderungen $(n=12.397)$, Andere $(n=12.340)$. 
Die nach Bildungsgruppen differenzierte Betrachtung bestätigt noch einmal, dass sich insbesondere junge Menschen auch für ebendiese engagieren. In Abbildung 11-14 ist dargestellt, dass 81,6 Prozent der engagierten Schülerinnen und Schülern sich für Kinder und Jugendliche einsetzen. Gut ein Viertel der Schülerinnen und
Schüler gibt an, sich für Familien zu engagieren, und immerhin 17,1 Prozent berichten über ein Engagement für ältere Menschen und 14,5 Prozent über ein Engagement für Menschen mit Migrationshintergrund. Die anderen Zielgruppen spielen für Schüler und Schülerinnen eine vergleichsweise geringe Rolle.

\section{Abbildung 11-14: Zielgruppen der freiwilligen Tätigkeit 2014, nach Bildung}

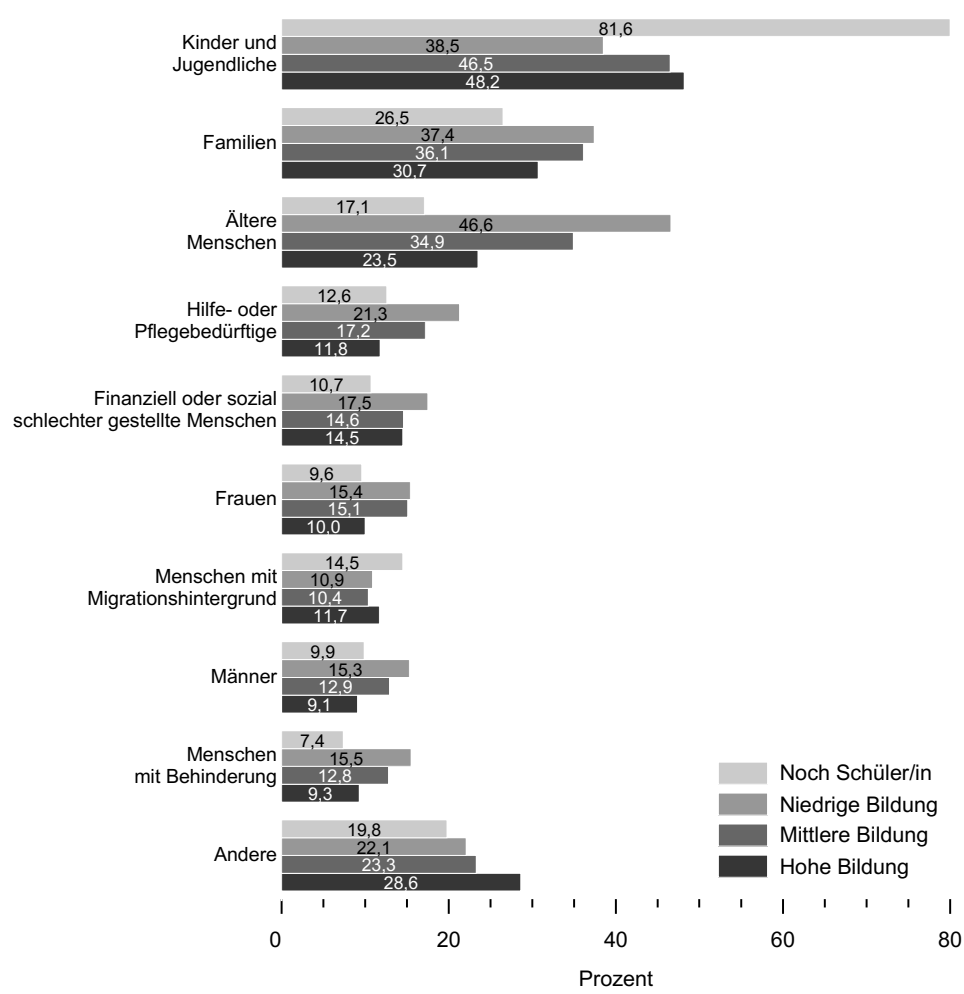

Quelle: FWS 2014, gewichtet, eigene Berechnungen (DZA). Basis: Alle Engagierten. Kinder und Jugendliche ( $n=12.398)$, Familien ( $n=12.374)$, Ältere Menschen $(n=12.378)$, Hilfe- oder Pflegebedürftige $(n=12.391)$, Finanziell oder sozial schlechter gestellte Menschen $(n=12.273)$, Frauen $(n=12.391)$, Menschen mit Migrationshintergrund $(n=12.366)$, Männer $(n=12.386)$, Menschen mit Behinderungen $(n=12.394)$, Andere $(n=12.337)$.

Interessant ist darüber hinaus, dass Engagierte mit hoher Bildung sich im Vergleich zu Engagierten mit niedriger oder mittlerer Bildung häufiger für Kinder und Jugendliche einsetzen. Ebenso geben von den Höhergebildeten vergleichsweise viele an, sich für eine andere, nicht näher definierte, Zielgruppe zu engagieren. Für die meisten anderen Zielgruppen engagieren sich Menschen mit mittlerer und vor allem niedriger Bildung anteilig häufiger als Menschen mit hoher Bildung. Insbesondere zeigt sich dies bei dem Engagement für ältere Menschen und bei dem Engagement für Hilfe- oder Pflegebedürftige. So berichten 46,6 Prozent der Engagierten 
mit niedriger Bildung, dass sie sich für ältere Menschen einsetzen, bei den Engagierten mit hoher Bildung liegt dieser Anteil lediglich bei 23,5 Prozent. Diese Bildungsunterschiede können allerdings auch auf die Alterszusammensetzung der Engagierten zurückzuführen sein. Denn wie bereits gezeigt wurde (Abbildung 1113), sind es insbesondere die älteren Engagierten, die sich für ältere Menschen einsetzen, und diese verfügen im Kohortenvergleich im Durchschnitt über eine niedrigere Bildung als die Jüngeren. Bei dem Engagement für Menschen mit Migrationshintergrund sowie bei dem Engagement für finanziell oder sozial schlechter gestellte Menschen zeigen sich vergleichsweise geringe Bildungsunterschiede.

\section{Abbildung 11-15: Kinder und Jugendliche als Zielgruppe des freiwilligen Engagements 2014, gesamt und nach Geschlecht}

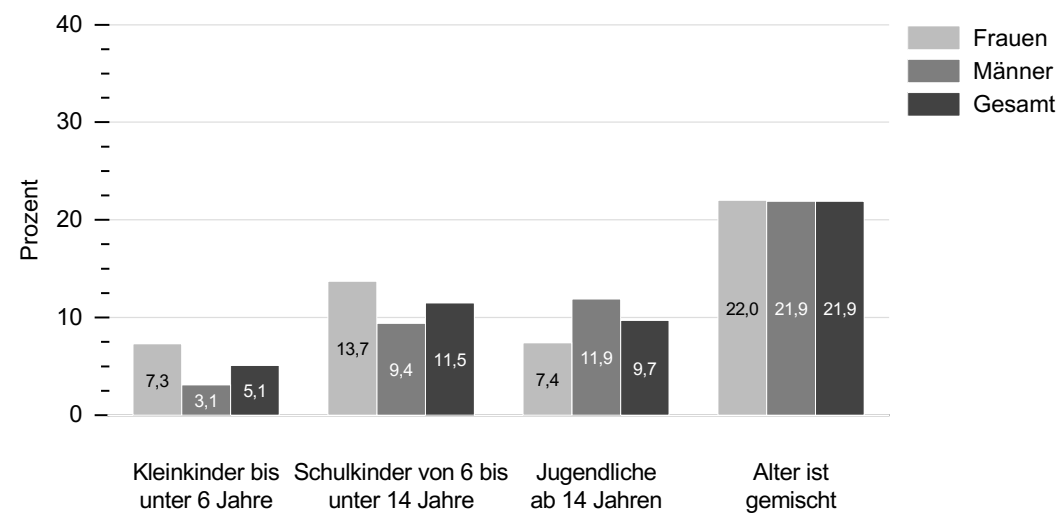

Quelle: FWS 2014, gewichtet, eigene Berechnungen (DZA). Basis: Alle Engagierten $(n=12.392$ ).

Betrachtet man die größte Zielgruppe der Kinder und Jugendlichen differenzierter, zeigen sich außerdem Geschlechterunterschiede (Abbildung 11-15). Während sich das Engagement von Frauen häufiger auf Kleinkinder und Schulkinder bis unter 14 Jahren richtet, engagieren sich Männer zu größeren Anteilen für Jugendliche ab 14 Jahren. Bei der überwiegenden Zahl von Personen, die sich für Kinder und Jugendliche engagieren, ist das Alter dieser Kinder und Jugendlichen allerdings gemischt. Dies trifft auf Frauen und Männer nahezu gleichermaßen zu. Differenziert man nach dem Alter der engagierten Frauen und Männer, zeigt sich, dass es insbesondere die 30- bis 49-jährigen Frauen sind, die sich für Kleinkinder bis unter sechs Jahren engagieren. Hier liegt die Vermutung nahe, dass die Lebensphase mit eigenen minderjährigen
Kindern - die heutzutage zumeist in dieses Alter fällt - das freiwillige Engagement in diesem Bereich auch begünstigt. Bei dem Engagement für Schulkinder von sechs bis unter 14 Jahren ist das höchste Engagement sowohl bei den 14- bis 29-jährigen sowie bei den 30 - bis 49 -jährigen Frauen zu beobachten. Bei dem Engagement für Jugendliche ab 14 Jahren dominieren hingegen die 14- bis 29-jährigen Männer (Tabelle 11-3 im Online-Anhang).

Die Lebensphase Alter umfasst heute einen zunehmend längeren Zeitraum mit unterschiedlichen Lebenssituationen. Während junge Alte im sechsten Lebensjahrzehnt häufig selbst noch aktiv und engagiert sind, ist das höhere Alter stärker von gesundheitlichen und funktionellen Einschränkungen geprägt. Dann treten mit höherem Alter beispielweise stärker pflegerische 
Versorgungs- und Unterstützungsleistungen durch Engagierte in den Vordergrund. Von Interesse ist es daher auch zu betrachten, an welche älteren Altersgruppen sich das freiwillige Engagement unterschiedlicher Personengruppen richtet. Am Beispiel von Menschen mit Demenz soll gezeigt werden, ob sich das Engagement auch an Personen mit spezifischen, chronischen Erkrankungen richtet.

\section{Abbildung 11-16: Ältere Menschen als Zielgruppe des freiwilligen Engagements 2014, gesamt und nach Geschlecht}

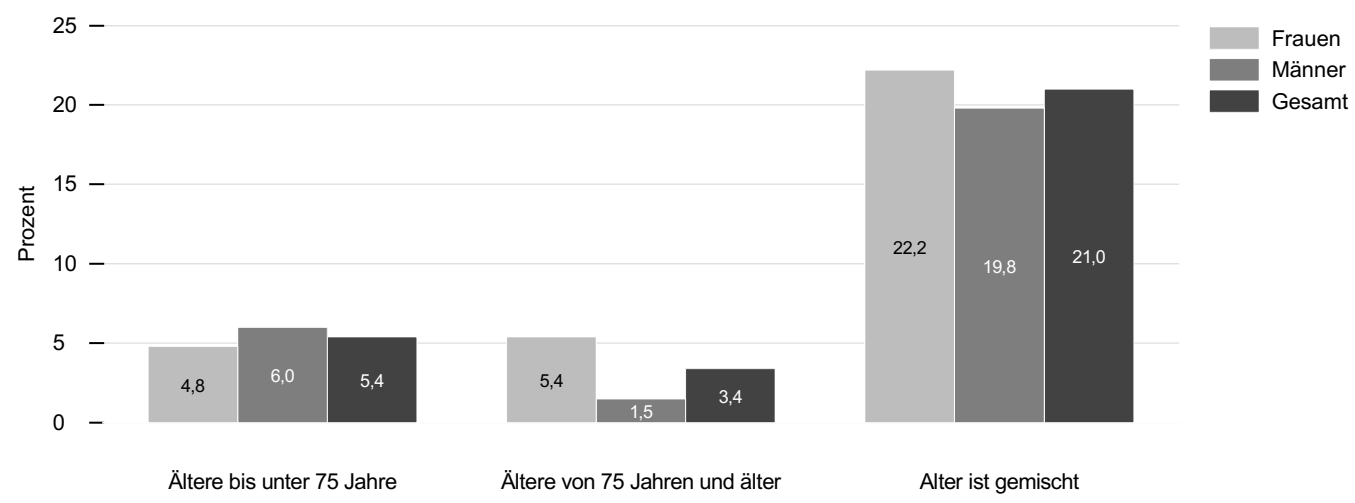

Quelle: FWS 2014, gewichtet, eigene Berechnungen (DZA). Basis: Alle Engagierten $(n=12.373$ ).

Dabei zeigt sich, dass die Zielgruppe der Älteren (ebenso wie bei Kindern und Jugendlichen) meist gemischten Alters ist. Es handelt sich also eher selten beispielsweise nur um hochaltrige Menschen, die unterstützt werden. 21,0 Prozent der Engagierten helfen mit ihrer Tätigkeit Älteren unterschiedlichen Alters, bei 5,4 Prozent der Engagierten richtet sich das Engagement auf Ältere bis unter 75 Jahren und nur bei 3,4 Prozent auf Personen ab 75 Jahren (Abbildung 11-16).
Dabei ist das Engagement von Männern etwas häufiger für jüngere Ältere (unter 75 Jahren), das Engagement von Frauen eher für Personen ab 75 Jahren. Engagierte höheren Alters engagieren sich insgesamt häufiger für Ältere; dies spiegelt sich auch bei der weiteren Altersdifferenzierung der Zielgruppen wieder: das heißt, Ältere engagieren sich häufiger als Jüngere für Ältere unter 75 Jahren, für Personen ab 75 Jahren und für Ältere gemischten Alters (Tabelle 11-4 im Online-Anhang). 
Personen, deren Engagement sich auf Ältere richtet, wurden zusätzlich gefragt, ob es sich bei diesen überwiegend um Menschen mit Demenz handelt. Insgesamt 2,8 Prozent aller Engagierten geben an, dass sich ihre freiwillige Tätigkeit auf diesen Personenkreis richtet, wobei der Anteil bei den engagierten Frauen mit 4,0 Prozent insgesamt etwas höher ist als bei den Männern (1,7 Prozent; Abbildung 11-17). Darüber hinaus zeigt sich, dass es insbesondere die Älteren sind, die ihr Engagement häufiger auf Menschen mit Demenz richten als jüngere Engagierte: 3,6 Prozent der 50- bis 64-jährigen Engagierten und 4,5 Prozent der Engagierten von 65 und mehr Jahren nennen Menschen mit Demenz als Zielgruppe ihres Engagements, aber nur 1,7 Prozent der 14- bis 29-jährigen und 2,0 Prozent der 30- bis 49-jährigen Engagierten (Abbildung 11-17). Dabei sind es vor allem Frauen im höheren Alter, deren freiwillige Tätigkeit Menschen mit Demenz zugutekommt. 7,3 Prozent der engagierten Frauen ab 65 Jahren und 5,5 Prozent der 50- bis 64-jährigen Frauen richten ihr Engagement auf Personen mit Demenz, gegenüber 2,8 Prozent der engagierten Frauen zwischen 30 und 49 Jahren und 1,3 Prozent derer zwischen 14 und 29 Jahren (Tabelle 11-5 im Online-Anhang).

\section{Abbildung 11-17: Menschen mit Demenz als Zielgruppe des freiwilligen Engagements 2014, gesamt sowie nach Geschlecht und nach Alter}

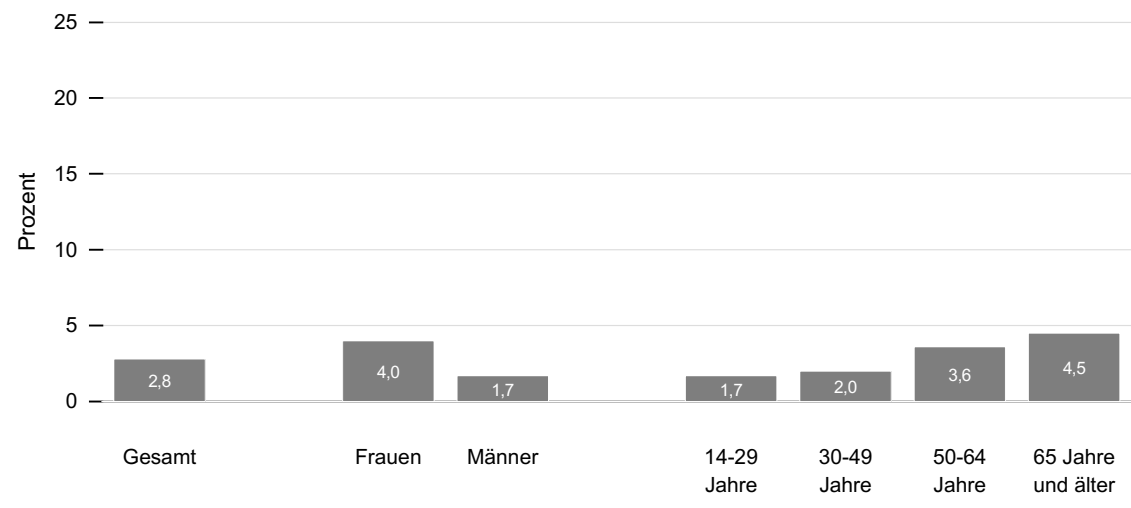

Quelle: FWS 2014, gewichtet, eigene Berechnungen (DZA). Basis: Alle Engagierten $(n=12.349)$.

Im Freiwilligensurvey 2014 wurde nicht nur nach der Zielgruppe, sondern auch nach der Zielregion des Engagements gefragt. Für die Zielregion zeigt sich eine deutliche Dominanz der Wohnregion: 89,1 Prozent der Engagierten richten ihre freiwillige Tätigkeit darauf (Tabelle 11-6 im Online-Anhang). Freiwilliges Engagement mit Bezug auf eine andere Region in Deutschland (7,6 Prozent) ist dagegen deutlich seltener, noch seltener richtet sich das Engagement auf eine andere Region in Europa (2,0 Prozent) oder auf ein Land oder eine Region außerhalb Europas (1,4 Prozent). Frauen engagieren sich mit 90,4 Prozent insge- samt etwas häufiger für die eigene Wohnregion als Männer (87,8 Prozent). Das Engagement von Personen zwischen 14 und 29 Jahren richtet sich mit 86,9 Prozent etwas seltener auf die eigene Wohnregion als das der übrigen Altersgruppen (Tabelle 11-6 im Online-Anhang). Allerdings trifft das auf die Gruppe der Schülerinnen und Schüler nicht zu: Hier engagieren sich mit 91,5 Prozent überdurchschnittlich viele der Engagierten für ihre eigene Wohnregion (Tabelle 11-7 im Online-Anhang). Nach Bildung betrachtet sind es vielmehr die Höhergebildeten, deren Engagement sich seltener auf die eigene Wohnregion 
richtet (86,4 Prozent), in dieser Bildungsgruppe findet sich auch etwas häufiger Engagement, das einer andere Region in Deutschland zugutekommen soll (9,4 Prozent vs. 7,6 Prozent über alle
Bildungsgruppen). Bei etwa einem Prozent der Engagierten richtet sich das Engagement ganz (0,7 Prozent) oder teilweise (0,3 Prozent) auf ein Entwicklungsland.

\subsection{Fazit}

Die Ergebnisse der hier präsentierten Daten des Freiwilligensurveys 2014 bestätigen noch einmal wie vielfältig die Tätigkeiten der engagierten Menschen in Deutschland sind. Hierbei dominieren die praktisch-organisatorischen Tätigkeiten. Die eher administrativen Arbeiten - wie Verwaltungstätigkeiten oderFundraising-werden hingegen deutlich seltener ausgeübt. Die Gewichtung einzelner Tätigkeitsinhalte unterscheidet sich zwischen Frauen und Männern nur wenig. Darüber hinaus ändern sich sowohl die generelle Rangfolge der Tätigkeitsinhalte als auch die Geschlechterunterschiede über die Zeit betrachtet kaum. Der Zeitvergleich zeigt jedoch, dass engagierte Personen zunehmend mehr Tätigkeitsinhalte angeben: In allen Tätigkeitsbereichen sind Zuwächse zu verzeichnen. Vor dem Hintergrund, dass sich das Zeitbudget für die freiwillige Tätigkeit nicht erhöht hat (siehe Kapitel 12), scheint die Tätigkeitsdichte im Engagement zugenommen zu haben. Dies kann eine Parallele zur Arbeitswelt darstellen, in der ähnliche Entwicklungen zu beobachten sind (Eichhorst, Kendzia, Schneider \& Buhlmann 2013). Im Bericht zum Freiwilligensurvey 2009 wurde in diesem Zusammenhang auch auf das gestiegene Selbstbewusstsein von Engagierten und eine damit verbundene anspruchsvollere Tätigkeitenbeschreibung hingewiesen (Gensicke 2015: 193). Insbesondere die Entwicklung der Engagementpolitik auf unterschiedlichen administrativen Ebenen sowie die verstärkte politische und gesellschaftliche Diskussion um freiwilliges und ehrenamtliches Engagement (Olk, Klein \& Hartnuß 2010; Hartnuß, Olk \& Klein 2011) könnten zu einer intensiveren Reflexion der eigenen Tätigkeit bei den Engagierten geführt haben, die sich in einer umfangreicheren und differenzierteren Beschreibung der eigenen freiwilligen Tätigkeit zeigt.

Der zu beobachtende Trend, dass Leitungsund Vorstandsfunktionen im Engagement zwar absolut gesehen nicht seltener geworden sind, anteilig innerhalb der Gruppe der Engagierten in den letzten fünfzehn Jahren jedoch abgenommen haben und sich dafür mehr Personen ohne Leitungs- und Vorstandsfunktion engagieren, kann ein Hinweis auf Veränderungen des Engagements und/oder dessen Organisationsformen sein. Die Ergebnisse des Kapitels 20 dieses Berichtes, in dem es um die organisationale Struktur des Engagement geht, weisen in die gleiche Richtung: Sie zeigen, dass freiwilliges Engagement zwar in allen Organisationsformen zugenommen hat, die größten Gewinne bei dem Engagement aber in individuell organisierten Gruppen zu verzeichnen sind. Aber nicht nur die Zunahme von Initiativen, Projekten oder selbstorganisierten Gruppen, die keine oder weniger formale hierarchische Strukturen und damit weniger Leitungs- und Vorstandspositionen aufweisen, kann zu einer Verschiebung innerhalb des Engagements und dessen Formen geführt haben. Auch eine Zunahme des Engagements in zentral gesteuerten Organisationen mit einem eher schmalen aber professionellen Management, wie beispielsweise Nichtregierungsorganisationen (NGOs) oder die Arbeit von Freiwilligenzentren könnte hierzu beigetragen haben - denn im Rahmen dieses Engagements gibt es für die freiwillig Tätigen meist weniger Möglichkeiten Leitungs- oder Vorstandsfunktionen zu übernehmen. Da die Bindungen an diese Organisationen häufig flexibler sind als die an 
traditionelle Vereine und sie somit eher mit den sich ändernden Anforderungen von Beruf und Familie vereinbar sind, könnte die Attraktivität dieser Engagementmöglichkeiten für bestimmte Bevölkerungsgruppen vergleichsweise hoch sein (Zimmer 2013).

Viele Engagierte nutzen das Internet im Rahmen ihrer freiwilligen Tätigkeiten, sei es zur Informationsbeschaffung, zum Austausch mit anderen oder um Informationen zu verbreiten. Nur eine kleine Gruppe Engagierter berichtet jedoch, die freiwillige Tätigkeit überwiegend oder gar ausschließlich über das Internet auszuüben. Rein internetbasiertes Engagement ist also quantitativ bislang eher eine Randerscheinung. Allerdings sollte nicht vergessen werden, dass diese Form des freiwilligen Engagements, selbst wenn sie von vergleichsweise wenigen Personen ausgeübt wird, aufgrund des großen Adressatenkreises, der über das Internet erreicht werden kann, dennoch eine hohe gesellschaftliche und politische Relevanz haben kann. Der etwas höhere Prozentsatz von ,Online Volunteers' bei den jüngeren Engagierten lässt zudem darauf schließen, dass diese Form des sich Engagierens zukünftig noch an Bedeutung gewinnen wird.

Obgleich sowohl bei Jüngeren als auch bei Älteren das Engagement in erster Linie der eigenen
Generation gilt, zeigen die Daten des Freiwilligensurveys, dass es im mittleren und insbesondere im höheren Lebensalter ein umfangreiches generationenübergreifendes Engagement gibt, das mit Bezug auf die Zielgruppe der Älteren zu größeren Teilen von Frauen geleistet wird. Darüber hinaus richtet sich das Engagement von Frauen häufiger auf hilfe- oder pflegebedürftige Personen. Dies steht in Einklang mit den Befunden zu informellen Unterstützungsleistungen innerhalb des sozialen Nahraums außerhalb der Familie (siehe Kapitel 9 und Kapitel 10). Auch hier sind es häufiger Frauen, die Menschen außerhalb des eigenen Haushaltes und der eigenen Familie pflegen oder betreuen. Möglicherweise ergeben sich über diese informellen Tätigkeiten auch Anstöße für freiwilliges Engagement in einem ähnlichen Tätigkeitsbereich.

Aufgrund der ansteigenden Zahlen von Flüchtlingen ist derzeit insbesondere mit einer Zunahme des Engagements für Migrantinnen und Migranten zu rechnen. Dabei ist allerdings noch offen, ob diese Entwicklung auch mit einem weiteren Anstieg der allgemeinen Engagementquoten einhergehen wird, oder aber, ob sich insbesondere Personen für diese Zielgruppe engagieren, die dies bereits in der Vergangenheit für eine andere Zielgruppe getan haben.

\section{Literatur}

Alscher, M., Droß, P. J., Priller, E., \& Schmeißer, C. (2013). Vereine an den Grenzen der Belastbarkeit. WZBrief Zivilengagement, 7, 2-7.

Blessing-Kapelke, U., \& Kaiser, F. (2013). "Attraktives Ehrenamt im Sport - Gesucht: Funktionsträger/innen in der 2. Lebenshälfte für Sportvereine“. Ein DOSB-Projekt zur vernetzten Ehrenamtsförderung, gefördert durch das Bundesministerium für Familie, Senioren, Frauen und Jugend, die Robert Bosch Stiftung und Generali Zukunfts Fonds. BBE-Newsletter, 15.

Eichhorst, W., Kendzia, M. J., Schneider, H., \& Buhlmann, F. (2013). Neue Anforderungen durch den Wandel der Arbeitswelt. Kurzexpertise für die Enquete-Kommission „Wachstum, Wohlstand, Lebensqualität" des Deutschen Bundestages. Bonn: IZA Forschungsinstitut zur Zukunft der Arbeit.

Eisnecker, P., \& Schupp, J. (2016). Flüchtlingszuwanderung: Mehrheit der Deutschen befürchtet negative Auswirkungen auf Wirtschaft und Gesellschaft. DIW Wochenbericht, 83(8), 158-164.

Gensicke, T. (2015). Freiwilliges Engagement in Deutschland: Freiwilligensurvey 2009. Wiesbaden: Springer VS. 
Gensicke, T., Picot, S., \& Geiss, S. (2006). Freiwilliges Engagement in Deutschland 1999-2004. Ergebnisse der repräsentativen Trenderhebung zu Ehrenamt, Freiwilligenarbeit und bürgerschaftlichem Engagement. Wiesbaden: VS Verlag für Sozialwissenschaften.

Hagen, C., \& Vogel, C. (2012). Freiwilliges und generationenübergreifendes Engagement von Frauen und Männern Analysepotenziale und Weiterentwicklung des Deutschen Freiwilligensurveys. Informationsdienst Altersfragen, 39(3), 3-9.

Hartnuß, B., Olk, T., \& Klein, A. (2011). Engagementpolitik. In: T. Olk \& B. Hartnuß (Hrsg.) Handbuch Bürgerschaftliches Engagement (S. 761-776). Weinheim: Beltz Juventa.

Jähnert, H. (2012). Was ist Online-Volunteering? BBE-Newsletter, 5.

Karakayali, S., \& Kleist, J. O. (2015). EFA-Studie. Strukturen und Motive der ehrenamtlichen Flüchtlingsarbeit (EFA) in Deutschland. Ergebnisse einer explorativen Umfrage vom November/Dezember 2014. Berlin: Berliner Institut für empirische Integrations- und Migrationsforschung der Humboldt-Universität zu Berlin (BIM).

Olk, T., Klein, A., \& Hartnuß, B. (Hrsg.) (2010). Engagementpolitik. Die Entwicklung der Zivilgesellschaft als politische Aufgabe. Wiesbaden: VS Verlag für Sozialwissenschaften.

Priller, E., Alscher, M., Droß, P. J., Paul, F., Poldrack, C. J., Schmeißer, C., \& Waitkus, N. (2012). Dritte-Sektor-Organisationen heute: Eigene Ansprüche und ökonomische Herausforderungen. Ergebnisse einer Organisationsbefragung (Discussion Paper SP IV 2012-402). Berlin: Wissenschaftszentrum Berlin für Sozialforschung.

Rosenbladt, B. von (Hrsg.) (2001). Freiwilliges Engagement in Deutschland. Freiwilligensurvey 1999. Ergebnisse der Repräsentativerhebung zu Ehrenamt, Freiwilligenarbeit und bürgerschaftlichem Engagement. Band 1: Gesamtbericht. Stuttgart: Kohlhammer.

Zimmer, A. (2013). Auslaufmodell Verein? Vom Veralten eines gesellschaftlichen Strukturmoments. Theorie und Praxis der sozialen Arbeit, 64(6), 447-455.

Open Access Dieses Kapitel wird unter der Creative Commons Namensnennung 4.0 International Lizenz (http://creativecommons.org/licenses/by/4.0/deed.de) veröffentlicht, welche die Nutzung, Vervielfältigung, Bearbeitung, Verbreitung und Wiedergabe in jeglichem Medium und Format erlaubt, sofern Sie den/die ursprünglichen Autor(en) und die Quelle ordnungsgemäß nennen, einen Link zur Creative Commons Lizenz beifügen und angeben, ob Änderungen vorgenommen wurden.

Die in diesem Kapitel enthaltenen Bilder und sonstiges Drittmaterial unterliegen ebenfalls der genannten Creative Commons Lizenz, sofern sich aus der Abbildungslegende nichts anderes ergibt. Sofern das betreffende Material nicht unter der genannten Creative Commons Lizenz steht und die betreffende Handlung nicht nach gesetzlichen Vorschriften erlaubt ist, ist für die oben aufgeführten Weiterverwendungen des Materials die Einwilligung des jeweiligen Rechteinhabers einzuholen. 